\title{
MONODROMY CONJECTURE FOR SOME SURFACE SINGULARITIES
}

\author{
BY E. ARTAL BARTOLO, P. CASSOU-NOGUÈS, I. LUENGO AND \\ A. MELLE HERNÁNDEZ
}

ABSTRACT. - In this work we give a formula for the local Denef-Loeser zeta function of a superisolated singularity of hypersurface in terms of the local Denef-Loeser zeta function of the singularities of its tangent cone. We prove the monodromy conjecture for some surfaces singularities. These results are applied to the study of rational arrangements of plane curves whose Euler-Poincaré characteristic is three.

(C) 2002 Éditions scientifiques et médicales Elsevier SAS

RÉSUMÉ. - Dans ce travail, nous donnons une formule pour la fonction zêta locale de Denef-Loeser d'une singularité superisolée d'hypersurface, en termes des fonctions zêta locales des singularités de son cône tangent. Nous démontrons la conjecture de la monodromie pour certaines singularités de surfaces. Nous appliquons ces résultats à l'étude d'arrangements, de caractéristique d'Euler trois, de courbes rationnelles.

(C) 2002 Éditions scientifiques et médicales Elsevier SAS

\section{Introduction}

Throughout this paper the complex numbers will be the ground field. The local Denef-Loeser zeta function $Z_{\text {top }, 0}(f, s) \in \mathbb{Q}(s)$ is an analytic (but not topological, see [4]) subtle invariant associated with any germ of an analytic function $f:\left(\mathbb{C}^{n+1}, 0\right) \rightarrow(\mathbb{C}, 0)$. This rational function was first introduced by J. Denef and F. Loeser as a sort of limit of the $p$-adic Igusa zeta function, see $[7,8]$ and it was called the topological zeta function. Its former definition was written in terms of any embedded resolution of its zero locus germ $(V, 0):=\left(f^{-1}(0), 0\right) \subset\left(\mathbb{C}^{n+1}, 0\right)$ (although it does not depend on any particular resolution). In [8], J. Denef and F. Loeser gave an intrinsic definition of $Z_{\text {top }, 0}(f, s)$ using arc spaces and the motivic zeta function - see also [10] and the Séminaire Bourbaki talk of E. Looijenga [24].

Let us recall the definition of the Denef-Loeser zeta functions associated with a polynomial $f \in \mathbb{C}\left[x_{0}, \ldots, x_{n}\right]$, see $[7,8]$. Let $\pi: Y \rightarrow \mathbb{C}^{n+1}$ be an embedded resolution of the hypersurface $V$ defined by the zero locus of $f$. Let $E_{i}, i \in I$, be the irreducible components of the divisor $\pi^{-1}\left(f^{-1}(0)\right)$. For each subset $J \subset I$ we set

$$
E_{J}:=\bigcap_{j \in J} E_{j} \quad \text { and } \quad \check{E}_{J}:=E_{J} \backslash \bigcup_{j \notin J} E_{J \cup\{j\}} .
$$

For each $j \in I$, let us denote by $N_{j}$ the multiplicity of $E_{j}$ in the divisor of $f \circ \pi$ and by $\nu_{j}-1$ the multiplicity of $E_{j}$ in the divisor of $\pi^{*}(\omega)$ where $\omega$ is a non-vanishing holomorphic $(n+1)$-form 
in $\mathbb{C}^{n+1}$. Then the local Denef-Loeser zeta function of $f$ is:

$$
Z_{\mathrm{top}, 0}(f, s):=\sum_{J \subset I} \chi\left(\check{E}_{J} \cap \pi^{-1}(0)\right) \prod_{j \in J} \frac{1}{\nu_{j}+N_{j} s} \in \mathbb{Q}(s),
$$

and the Denef-Loeser zeta function of $f$ is:

$$
Z_{\mathrm{top}}(f, s):=\sum_{J \subset I} \chi\left(\check{E}_{J}\right) \prod_{j \in J} \frac{1}{\nu_{j}+N_{j} s} \in \mathbb{Q}(s),
$$

where $\chi$ denotes the Euler-Poincaré characteristic.

Each exceptional divisor of an embedded resolution $\pi:(Y, \mathcal{D}) \rightarrow\left(\mathbb{C}^{n+1}, 0\right)$ of the germ $(V, 0)$ gives a candidate pole of the rational function $Z_{\mathrm{top}, 0}(f, s)$. Nevertheless only a few of them give an actual pole of $Z_{\text {top }, 0}(f, s)$. There are several conjectures related to the Denef-Loeser zeta functions. In this paper we are interested in the monodromy conjecture, see [6,7].

It is known that, for any given $x \in V$, the Milnor fibration of the holomorphic function $f$ at $x$ is the $C^{\infty}$ locally trivial fibration $f \mid: B_{\varepsilon}(x) \cap f^{-1}\left(\mathbb{D}_{\eta}^{*}\right) \rightarrow \mathbb{D}_{\eta}^{*}$, where $B_{\varepsilon}(x)$ is the open ball of radius $\varepsilon$ centered at $x, \mathbb{D}_{\eta}=\{z \in \mathbb{C}:|z|<\eta\}$ and $\mathbb{D}_{\eta}^{*}$ is the open punctured disk $\left(0<\eta \ll \varepsilon\right.$ and $\varepsilon$ small enough). Any fiber $F_{f, x}$ of this fibration is the Milnor fiber of $f$ at $x$. The monodromy transformation $h: F_{f, x} \rightarrow F_{f, x}$ is the well-defined (up to isotopy) diffeomorphism of $F_{f, x}$ induced by a small loop around $0 \in \mathbb{D}_{\eta}$. The complex algebraic monodromy of $f$ at $x$ is the corresponding linear transformation $h_{*}: H_{*}\left(F_{f, x}, \mathbb{C}\right) \rightarrow H_{*}\left(F_{f, x}, \mathbb{C}\right)$ on the homology groups.

The local monodromy conjecture states that if $s_{0}$ is a pole of the Denef-Loeser zeta function $Z_{\mathrm{top}, 0}(f, s)$ of the local singularity defined by $f$, then $\exp \left(2 \mathrm{i} \pi s_{0}\right)$ is an eigenvalue of the local monodromy at some complex point of $f^{-1}(0)$. Note that if $f$ defines an isolated hypersurface singularity, then $\exp \left(2 \mathrm{i} \pi s_{0}\right)$ has to be an eigenvalue of the complex algebraic monodromy of the germ $\left(f^{-1}(0), 0\right)$.

There are three general problems to consider when trying to prove (or disprove) the conjecture using resolution of singularities:

(i) Explicit computation of an embedded resolution of the hypersurface $(V, 0) \subset\left(\mathbb{C}^{n+1}, 0\right)$.

(ii) Elimination of the candidate poles which are not actually poles of $Z_{\mathrm{top}, 0}(f, s)$.

(iii) Explicit computation of the eigenvalues of the complex algebraic monodromy (or computing the characteristic polynomials of the corresponding action of the complex algebraic monodromy) in terms of the resolution data.

The monodromy conjecture, which was first stated for the Igusa zeta function, has been proved for curve singularities by F. Loeser [21]. F. Loeser actually proved a stronger version of the monodromy conjecture: that any pole of the Denef-Loeser zeta function gives a root of the Bernstein polynomial of the singularity. The behavior of the Denef-Loeser zeta function for germs of curves is rather well understood once an explicit embedded resolution $\pi:(Y, \mathcal{D}) \rightarrow\left(\mathbb{C}^{2}, 0\right)$ of curve singularities is known, e.g. the minimal one. Basically any irreducible component $E$ of the exceptional divisor $\mathcal{D}=\pi^{-1}(0)$ which intersects the total transform $\pi^{-1}(V)$ in at most two points has no contribution to the residue of $Z_{\mathrm{top}, 0}(f, s)$ at the candidate pole. This was proved in consecutive works by Strauss, Meuser, Igusa and Loeser for Igusa's local zeta function, but the same the proof works for the Denef-Loeser zeta function. W. Veys later gave a much simpler and more conceptual proof of this in [31] and proved in [30] that all other $E$ actually do give poles.

There are other classes of singularities where the embedded resolution is known. For example, for any singularity of hypersurface defined by an analytic function which is non-degenerated with 
respect to its Newton polytope, problems (i) and (iii) above are solved. Nevertheless the problem (ii) seems to be a hard combinatorial problem. This problem was partially solved by F. Loeser in the case where $f$ has a non-degenerate Newton polytope and satisfies certain extra technical conditions [22].

An embedded resolution is also known for superisolated surface singularities, SIS for short - see [2]. Singularities of this type, named by I. Luengo in [25], were used to prove that the $\mu$-constant stratum of an isolated hypersurface singularity is not smooth - see also [28]. E. Artal used them to disprove a conjecture of S.S.T. Yau.

Even in one of the simplest cases where $f$ has non-isolated singularities, namely the case of homogeneous surfaces, problems (i) and (iii) are solved, but problem (ii) was still open. For any degree $d$ and any homogeneous polynomial $f_{d} \in \mathbb{C}\left[x_{1}, x_{2}, x_{3}\right]$ a candidate pole is $s_{0}=-3 / d$. It can be seen when one blows up once at the origin. A sufficient condition for the candidate pole $s_{0}=-3 / d$ of $Z_{\mathrm{top}, 0}(f, s)$ to verify the monodromy conjecture is the following: $\chi\left(\mathbb{P}^{2} \backslash\left\{f_{d}=0\right\}\right) \neq 0$.

B. Rodrigues and W. Veys proved in [26] the monodromy conjecture for any homogeneous polynomial $f_{d} \in \mathbb{C}\left[x_{1}, x_{2}, x_{3}\right]$ satisfying $\chi\left(\mathbb{P}^{2} \backslash\left\{f_{d}=0\right\}\right) \neq 0$. They excluded the case $\chi\left(\mathbb{P}^{2} \backslash\left\{f_{d}=0\right\}\right)=0$ because they couldn't solve problem (ii) for the candidate pole $s_{0}=-3 / d$.

In this paper we prove the monodromy conjecture for SIS singularities and also complete the proof of the monodromy conjecture for homogeneous polynomials in three variables. More precisely, the results of this paper are the following.

Let $f$ be a germ of a superisolated hypersurface singularity defined by

$$
f=f_{d}+f_{d+1}+\cdots \in \mathbb{C}\left\{x_{0}, x_{1}, \ldots, x_{n}\right\} .
$$

Let us denote by $C_{m} \subset \mathbb{P}^{n}$ the divisor associated with the homogeneous polynomial $f_{m}$. By definition, the hypersurface singularity $(V, 0)=\left(f^{-1}(0), 0\right) \subset\left(\mathbb{C}^{n+1}, 0\right)$ is superisolated, SIH for short, if and only if the projective set $C_{d+1} \cap \operatorname{Sing}\left(C_{d}\right)$ is empty. This is equivalent to the fact that one needs to blow up the origin only once to resolve the singularity. For each $P \in \operatorname{Sing}\left(C_{d}\right)$ we choose analytic coordinates centered at the origin and we denote by $g^{P}$ the equation of $C_{d}$ in these coordinates.

Our first goal is to obtain a formula for the Denef-Loeser zeta function of a SIH singularity in terms of similar invariants of its tangent cone. Such a formula is given in Section 1.

COROLlary 1.12. - Let $f:=f_{d}+f_{d+1}+\cdots \in \mathbb{C}\left\{x_{0}, x_{1}, \ldots, x_{n}\right\}$ define a SIH singularity $(V, 0) \subset\left(\mathbb{C}^{n+1}, 0\right)$. Then its local Denef-Loeser zeta function satisfies the following equality

$$
\begin{aligned}
Z_{\mathrm{top}, 0}(V, s)= & \frac{\chi\left(\mathbb{P}^{n} \backslash C_{d}\right)}{t-s}+\frac{\chi\left(\check{C}_{d}\right)}{(t-s)(s+1)} \\
& +\sum_{P \in \operatorname{Sing}\left(C_{d}\right)}\left(\frac{1}{t}+(t+1)\left(\frac{1}{(t-s)(s+1)}-\frac{1}{t}\right) Z_{\mathrm{top}, 0}\left(g^{P}, t\right)\right),
\end{aligned}
$$

where $t:=n+1+(d+1) s, \check{C}_{d}=C_{d} \backslash \operatorname{Sing}\left(C_{d}\right)$ and $Z_{\mathrm{top}, 0}\left(g^{P}, s\right)$ stands for the local DenefLoeser zeta function for the germ $g^{P}$ at the singular point $P \in \operatorname{Sing}\left(C_{d}\right)$.

Using the formula above, the characteristic polynomial formula of the complex algebraic monodromy of a SIS singularity - e.g. see [2] - and the monodromy conjecture for curves see $[21]$ - we prove the following for a SIS singularity of multiplicity $d$ :

- If $\chi\left(\mathbb{P}^{2} \backslash C_{d}\right)>0$ then the monodromy conjecture holds for $(V, 0) \subset\left(\mathbb{C}^{3}, 0\right)$.

- If $\chi\left(\mathbb{P}^{2} \backslash C_{d}\right) \leqslant 0$, then every pole of the local Denef-Loeser zeta function of $(V, 0) \subset\left(\mathbb{C}^{3}, 0\right)$, except for $s_{0}=-3 / d$, verifies the monodromy conjecture. 
Furthermore, if $\chi\left(\mathbb{P}^{2} \backslash C_{d}\right)=0$, and $s_{0}=-3 / d$ is a pole of the local Denef-Loeser zeta function of the plane curve germ $C_{d}$ at some singular point, then the monodromy conjecture for $(V, 0) \subset\left(\mathbb{C}^{3}, 0\right)$ also holds.

We split the study of the remaining cases in two parts.

We say that a degree $d$ effective divisor $D$ on $\mathbb{P}^{2}(d>3)$ is a bad divisor if $\chi\left(\mathbb{P}^{2} \backslash D\right) \leqslant 0$ and $s_{0}=-3 / d$ is not a pole of $Z_{\text {top }, P}\left(g_{D}^{P}, s\right)$ for any singular point $P$ in its support $D_{\text {red }}$, where $g_{D}^{P}$ is the local equation of the divisor $D$ at $P$.

Let us define

$$
\rho\left(C_{d}\right):=\chi\left(\mathbb{P}^{2} \backslash C_{d}\right)+\chi\left(\check{C}_{d}\right) \frac{d}{d-3}+\sum_{P \in \operatorname{Sing}\left(C_{d}\right)} Z_{\mathrm{top}, P}\left(g^{P},-\frac{3}{d}\right) \in \mathbb{Q} .
$$

When the tangent cone $C_{d}$ is a bad divisor, $s_{0}=-3 / d$ is a simple pole of $Z_{\mathrm{top}, 0}(f, s)$ if and only if $\rho\left(C_{d}\right) \neq 0$.

Next we study the bad divisors $C_{d}$ such that $\rho\left(C_{d}\right) \neq 0$. It turns out that the residue $\rho\left(C_{d}\right)$ agrees with the value of $z\left(C_{d}, s\right)$ at $s_{0}=-3 / d$, where $z(D, s)$ stands for the Denef-Loeser zeta function associated with a divisor $D$ on $\mathbb{P}^{2}$. This invariant was recently introduced by W. Veys see [33]. This residue also has another meaning: $\rho\left(C_{d}\right)$ coincides with an invariant $\zeta_{K}$ associated with the $\mathbb{Q}$-canonical divisor $K:=(-3 / d) C_{d}$ on the rational surface $\mathbb{P}^{2}$. In this paper we use both of these meanings to extend the notion of the residue $\rho(D)$ to bad divisors $D$ on $\mathbb{P}^{2}$ (not only for reduced curves $C_{d}$ ) and to some canonical divisors on rational surfaces.

The main part of Section 2 is devoted to determining bad divisors $D$ on $\mathbb{P}^{2}$ such that $\rho(D) \neq 0$. Note that the Euler-Poincaré characteristic condition on a bad divisor $D$ implies that $D$ has at least two irreducible components, all of them rational curves - see [16,15,19,5].

Our second main result is the following theorem.

THEOREM 2.15. - Let $D$ be a bad divisor on $\mathbb{P}^{2}$. If $\rho(D) \neq 0$, then the irreducible components of $D$ are in a pencil $\Lambda$ of rational curves having only one base point and such that any fiber minus the base point is isomorphic to $\mathbb{C}$. Moreover, at least one (resp. two) generic fiber of $\Lambda$ is contained in $D$ if the pencil has two exceptional fibers (resp. one).

The proof of this result is quite elaborate. We use the following result by W. Veys [32].

Veys' TheOREM. - Let $D$ be a curve in $\mathbb{P}^{2}$. If $\chi\left(\mathbb{P}^{2} \backslash D\right) \leqslant 0$, then $D$ can be extended to a configuration $D^{\prime} \supset D$, still satisfying $\chi\left(\mathbb{P}^{2} \backslash D^{\prime}\right) \leqslant 0$, for which there exists a diagram

$$
\Sigma \stackrel{g}{\longleftarrow} X \stackrel{f}{\longrightarrow} \mathbb{P}^{2},
$$

where $\Sigma$ is a ruled surface, $f$ is a composition of blowing-ups with center in $D^{\prime}$, and $g$ is a composition of blowing-downs whose exceptional curve is contained in $f^{-1}\left(D^{\prime}\right)$. Moreover, one can require the configuration $g\left(f^{-1}\left(D^{\prime}\right)\right)$ to consist of one of the following:

(A) One section $C_{1}$ and at least two fibers, or

(B) Two disjoint sections $C_{1}$ and $C_{2}$ and at least one fiber.

The proof of Theorem 2.15 entails the study of the behavior of the invariant $\zeta_{K}$ when applying blow-up and blow-down processes. This step has been partially studied in [29] in a slightly different context.

Only one type of blow-up has an effect on $\zeta_{K}$. If one starts with a bad divisor $D$ of degree $d$ in $\mathbb{P}^{2}$, the canonical divisor $(-3 / d) D$ is transformed by Veys' process into a canonical divisor on the ruled surface $\Sigma$ with support on a curve of type (A) or (B). It is easily seen that such a 
canonical divisor $K_{\Sigma}$ in a ruled surface verifies $\zeta_{K_{\Sigma}}=0$. Then we show that arrangements of rational curves in which the residue changes can be put in a rational pencil of type $(0,1)$ on $\mathbb{P}^{2}$ (see Appendix A).

In order to complete the proof of the monodromy conjecture for SIS singularities we have to deal with singularities having a tangent cone $C_{d}$ which is a bad divisor with $\rho\left(C_{d}\right) \neq 0$. The last step in the proof consists of computing the Alexander polynomial of the curves $C_{d}$ satisfying the properties described in Theorem 2.15. For this purpose we use Kashiwara's classification [17] of pencils of rational curves of type $(0,1)$. We prove, case by case, that $\exp (2 \mathrm{i} \pi(-3 / d))$ is a root of the Alexander polynomial of the curve at its only singular point with the required multiplicity. Finally, using the computations in [2], we have that $\exp (2 \mathrm{i} \pi(-3 / d))$ is a root of the Alexander polynomial of the corresponding SIS singularity.

This work also allows for the generalization of the proof given by B. Rodrigues and W. Veys of the monodromy conjecture to the case of homogeneous polynomials $f_{d} \in \mathbb{C}\left[x_{1}, x_{2}, x_{3}\right]$ with $\chi\left(\mathbb{P}^{2} \backslash\left\{f_{d}=0\right\}\right)=0$.

In the case of curves, if an exceptional divisor $E_{i}$ satisfies $\chi\left(\check{E}_{i}\right)=0\left(\check{E}_{i}=E_{i} \backslash \bigcup_{j \neq i} E_{j}\right)$ then $E_{i}$ does not contribute to the candidate pole $\frac{-\nu_{i}}{N_{i}}$ of $Z_{\text {top }, 0}(f, s)$. This question is more complicated in the case of surfaces. W. Veys proved in [29] for many such configurations that $E$ does not contribute to the candidate pole $-\nu / N$, assuming that $E$ doesn't intersect any other component with the same ratio of numerical data (this is the general case).

In this work we find that some candidate poles which appear only on exceptional divisors $E_{i}$ of the resolution verifying $\chi\left(\check{E}_{i}\right)=0$ are actual poles of the Denef-Loeser zeta function. This is the case for the first exceptional component of the resolution of a SIS singularity or a homogeneous surface whose tangent cone $D$ is a bad divisor with residue $\rho(D) \neq 0$ at the pole $-3 / d$.

In our opinion, the theorems we prove in this paper give strong evidence for the monodromy conjecture in the following sense. We prove that for bad divisors $D, \exp (2 \mathrm{i} \pi(-3 / d))$ is an eigenvalue of the monodromy of the only singular point of $D_{\text {red }}$. This fact is not evident a priori - see Section 5 for details, where we also study arrangements of rational curves on $\mathbb{P}^{2}$.

Finally we use an example of a bad divisor $D$ on $\mathbb{P}^{2}$ whose residue $\rho(D) \neq 0$ to answer an open question of D. Siersma - see Section 6 for details.

\section{General formula for the Denef-Loeser zeta function}

The definition of the Denef-Loeser zeta function associated with a morphism $f: X \rightarrow \mathbb{C}$ can be extended to an effective divisor $D$ on a nonsingular $(n+1)$-dimensional complex variety $X$ - see [33]. If $\pi: Y \rightarrow X$ is an embedded resolution of the support of $D$, and $E_{i}, i \in I$, are the irreducible components of the divisor $\pi^{-1}(\operatorname{Supp} D)$ with associated multiplicities $N_{i}, i \in I$, where $\pi^{*}(D)=\sum N_{i} E_{i}$. Let $\omega$ be a local generator of the sheaf of holomorphic $(n+1)$-forms on $X$ and let $\operatorname{div}\left(\pi^{*} \omega\right)=\sum\left(\nu_{i}-1\right) E_{i}$ be the divisor of its pull-back. The Denef-Loeser zeta function of $D$ is defined by

$$
z(D, s):=\sum_{J \subset I} \chi\left(\check{E}_{J}\right) \prod_{j \in J} \frac{1}{\nu_{j}+N_{j} s} \in \mathbb{Q}(s) .
$$

We shall compute the Denef-Loeser zeta function of a SIH singularity in $\mathbb{C}^{n+1}$. We will make use of three general principles which, at least implicitly, are well known. We begin by recalling the generalization of this zeta function by J. Denef and F. Loeser [8,33].

Let $X$ be an algebraic $(n+1)$-manifold, $f: X \rightarrow \mathbb{C}$ an algebraic function and $\omega$ an $(n+1)$-meromorphic differential form (algebraically defined) on $X$ such that the polar locus 
of $\omega$ is included in the zero locus of $f$. Algebraic can be replaced by analytic either in the germ case or by adding some natural hypothesis about finiteness. Thus the Denef-Loeser zeta function $Z_{\text {top }}(f, \omega, s)$ can be defined analogously. In this case, the $\nu$-invariant is associated with the form $\omega$ rather than with a non-vanishing form. We state the three main principles:

PBM PRINCIPLE 1.1 (See [33, Theorem 5.6]).- Let $\pi: V \rightarrow X$ be a proper birational morphism. Then

$$
Z_{\text {top }}(f, \omega, s)=Z_{\text {top }}\left(f \circ \pi, \pi^{*}(\omega), s\right) .
$$

STRATUM PRINCIPLE 1.2. - Let $X=\coprod_{S \in \mathcal{S}} S$ be a finite prestratification of $X$ such that for each $x \in X$, the local Denef-Loeser zeta function $Z_{\mathrm{top}, x}(f, \omega, s)$ at $x$, depends only on the stratum $S$ containing $x$. Let us denote by $Z_{\mathrm{top}, S}(f, \omega, s)$ the common zeta function associated with the stratum $S$. Then,

$$
Z_{\mathrm{top}}(f, \omega, s)=\sum_{S \in \mathcal{S}} \chi(S) Z_{\mathrm{top}, S}(f, \omega, s) .
$$

The key point in this principle is that one may construct a resolution for both $f$ and $\omega$, such that one can rearrange the terms on the left-hand side of the formula to fit the right-hand side of the formula.

FUBINI'S PRINCIPLE 1.3. - Let us consider two germs of function $f_{i}:\left(\mathbb{C}^{n_{i}+1}, 0\right) \rightarrow(\mathbb{C}, 0)$ and two germs of $\left(n_{i}+1\right)$-holomorphic form $\omega_{i}, i=1,2$. We consider $f:=f_{1} f_{2}$ and $\omega:=\omega_{1} \wedge \omega_{2}$ as germs of function and form in $\left(\mathbb{C}^{n_{1}+n_{2}+2}, 0\right)$. Then,

$$
Z_{\mathrm{top}, 0}(f, \omega, s)=\prod_{i=1}^{2} Z_{\mathrm{top}, 0}\left(f_{i}, \omega_{i}, s\right) .
$$

In order to prove Fubini's Principle, it is enough to consider a proper birational mapping obtained by combining the resolution of $f_{1}$ and $\omega_{1}$ in the first variables and the identity in the second variables. The PBM Principle assures the invariance of the Denef-Loeser zeta function. Thus, we obtain a prestratification such that for any stratum, the pull-back of $f$ can be written as some power of coordinate functions in the first variables, and $f_{2}$ and $\omega_{2}$ in the second variables. On each stratum, we now consider the proper birational mapping associated with the second variables and the result easily follows.

Example 1.4. - Let us take a germ $f:\left(\mathbb{C}^{n+1}, 0\right) \rightarrow(\mathbb{C}, 0)$ and a germ of holomorphic form $\omega$. We can choose a good representative $W$ (where $f$ and $\omega$ are defined). $W$ comes with a finite prestratification as in (1.2). All the strata except the origin have Euler-Poincare characteristic zero. Then the zeta function of the germ is the same as the zeta function of the good representative.

Example 1.5. - Let us take a germ $f:\left(\mathbb{C}^{n+1}, 0\right) \rightarrow(\mathbb{C}, 0)$ and a germ of holomorphic form $\omega$. Fix a good representative $W$ where $f$ and $\omega$ are defined. Let us consider the blowing-up $\pi: \hat{W} \rightarrow W$ along a smooth subvariety of $W$ containing 0 . Consider $D:=\pi^{-1}(0)$ and let $\mathcal{S}_{D}$ be a finite prestratification of $D$ satisfying the property 1.2 . Then,

$$
Z_{\mathrm{top}, 0}(f, \omega, s)=\sum_{S \in \mathcal{S}_{D}} \chi(S) Z_{\mathrm{top}, S}\left(f \circ \pi, \pi^{*}(\omega), s\right) .
$$


We apply these principles to a SIH singularity defined by

$$
f:=f_{d}+f_{d+1}+\cdots \in \mathbb{C}\left\{y_{0}, y_{1}, \ldots, y_{n}\right\} .
$$

By definition, the projective set $C_{d+1} \cap \operatorname{Sing}\left(C_{d}\right)$ is empty. Therefore, the set $\operatorname{Sing}\left(C_{d}\right)$ of singular points of the projective hypersurface $C_{d}$ is finite. Let us denote by $\check{C}_{d}$ the regular part of $C_{d} \subset \mathbb{P}^{n}$. Let $\pi: V \rightarrow\left(\mathbb{C}^{n+1}, 0\right)$ be the blow-up at the origin in $\mathbb{C}^{n+1}$. Let $\varphi$ be the lifting of $f$ to $V$. Let us consider the stratification of the exceptional divisor $E \simeq \mathbb{P}^{n}$ of this blowing-up into the following strata:

(a) 0-dimensional strata $S_{i}^{0}(i=1, \ldots, s)$ each consisting of one point $P_{i} \in \operatorname{Sing}\left(C_{d}\right)$;

(b) $(n-1)$-dimensional stratum $S^{n-1}=\check{C}_{d}$;

(c) $n$-dimensional stratum $S^{n}=\mathbb{P}^{n} \backslash C_{d}$.

In a neighborhood of the point $P \in \operatorname{Sing}\left(C_{d}\right)$ in $V$, there exists a coordinate system $z, x_{1}, \ldots, x_{n}$ such that (locally) $\mathbb{P}^{n}$ coincides with the hyperplane $\{z=0\}$ and the function $f \circ \pi$ has the form $v \cdot z^{d} \cdot\left(g^{P}\left(x_{1}, \ldots, x_{n}\right)+z\right)$, where $v$ is invertible $(v(0) \neq 0)$ (from now on, we will simply omit such "non-essential" factors), $g^{P}:\left(\mathbb{C}^{n}, 0\right) \rightarrow(\mathbb{C}, 0)$ is a germ of an analytic function whose zero locus coincides with the germ of the hypersurface $C_{d}$ at $P$.

In the same way, at each point of $S^{n-1}$ there exists a local system of coordinates such that the lifting $\varphi$ has the normal form $z^{d} \cdot x_{1}$. Finally, at each point of $S^{n}$ the lifting $\varphi$ has the normal form $z^{d}$ for some local coordinates. Let $\omega:=d x_{1} \wedge \cdots \wedge d x_{n} \wedge d z$, then in the corresponding coordinates the pull-back $\pi^{*}\left(d y_{0} \wedge \cdots \wedge d y_{n}\right)$ is $z^{n} \omega$.

Then from the PBM and Stratum Principles one gets

$$
\begin{aligned}
Z_{\mathrm{top}, 0}(f, s)= & \frac{\chi\left(\mathbb{P}^{n} \backslash C_{d}\right)}{t-s}+\frac{\chi\left(\check{C}_{d}\right)}{(t-s)(s+1)} \\
& +\sum_{P \in \operatorname{Sing}\left(C_{d}\right)} Z_{\mathrm{top}, P}\left(\left(z-g^{P}\left(x_{1}, \ldots, x_{n}\right)\right) z^{d}, z^{n} \omega, s\right),
\end{aligned}
$$

where $t:=n+1+(d+1) s$. Let us fix a singular point $P$ and set $g:=g^{P}$. Recall that in the local coordinates $x_{1}, \ldots, x_{n}, z$ the point $P$ is the origin, and $z=0$ is the equation of the exceptional divisor. The main point is to consider a birational map $h_{1}$ which is both an embedded resolution of $g^{-1}(0)$ in coordinates $x_{1}, \ldots, x_{n}$ and the identity in $z$.

Let us fix some notation about embedded resolutions $h: Y \rightarrow\left(\mathbb{C}^{n}, 0\right)$ of $g^{-1}(0)$. With each irreducible component $D_{0}, D_{1}, \ldots, D_{r}$ of the total transform of $g^{-1}(0)$, we associate the numbers $N_{i}$ and $\nu_{i}$, as usual. For each subset $J \subset\{0,1, \ldots, r\}$, we define the number $\chi_{J}:=\chi\left(\check{D}_{J} \cap h^{-1}(0)\right)$, where $D_{J}$ and $\check{D}_{J}$ are defined as in the introduction. Then

$$
Z_{\mathrm{top}, 0}(g, s)=\sum_{J \subset\{0,1, \ldots, r\}} \chi_{J} \prod_{i \in J} \frac{1}{\nu_{i}+N_{i} s} .
$$

The following formula can be easily deduced from the first two principles:

$$
Z_{\mathrm{top}, 0}\left(\left(z-g\left(x_{1}, \ldots, x_{n}\right)\right) z^{d}, z^{n} \omega, s\right)=\sum_{J \subset\{0,1, \ldots, r\}} \chi_{J} Z_{\mathrm{top}, 0}\left(\left(z-x^{J}\right) z^{d}, z^{n} \omega^{J}, s\right),
$$

where if $J=\left\{j_{1}, \ldots, j_{l}\right\}$, then

$$
x^{J}:=\prod_{k=1}^{l} x_{k}^{N_{j_{k}}}, \quad \omega^{J}:=\left(\prod_{k=1}^{l} x_{k}^{\nu_{j_{k}}-1}\right) d x_{1} \wedge \cdots \wedge d x_{n} \wedge d z
$$


Notation 1.6. - Given $n_{j}:=a_{j}+b_{j} s, a_{j}, b_{j} \in \mathbb{Z}_{>0}, j=1, \ldots, l$ and $m_{1}, \ldots, m_{l} \in \mathbb{Z}_{>0}$, $\tau:=a s+b, a, b \in \mathbb{Z}_{>0}$, we denote the local Denef-Loeser zeta function associated with the germs of function $q z^{a} h$ and of form $z^{b} \eta$ by

$$
Z\left(n_{1}, \ldots, n_{l} ; m_{1}, \ldots, m_{l} ; \tau, s\right):=Z_{\mathrm{top}, 0}\left(q z^{a} h, z^{b} \eta, s\right),
$$

where

$$
q:=\prod_{k=1}^{l} x_{k}^{b_{k}}, \quad h:=z-\prod_{k=1}^{l} x_{k}^{m_{k}}, \quad \eta:=\left(\prod_{k=1}^{l} x_{k}^{a_{k}-1}\right) d x_{1} \wedge \cdots \wedge d x_{n} \wedge d z .
$$

We next compute this local Denef-Loeser zeta function by blowing up along the coordinate subspace $z=x_{l}=0$ and applying the above mentioned principles.

Formula 1.7. - Let us assume that $l>1$. If $m_{l}>1$, then using the PBM and Stratum Principles one gets

$$
\begin{aligned}
\left(n_{1}, \ldots, n_{l-1}, n_{l} ; m_{1}, \ldots, m_{l-1}, m_{l} ; \tau, s\right) \\
=\left(\prod_{j=1}^{l} \frac{1}{n_{j}}\right) \frac{1}{n_{l}+\tau+s+1} \\
\quad+Z\left(n_{1}, \ldots, n_{l-1}, n_{l}+\tau+s+1 ; m_{1}, \ldots, m_{l-1}, m_{l}-1 ; \tau, s\right),
\end{aligned}
$$

and using Fubini's Principle

$$
\begin{aligned}
Z & \left(n_{1}, \ldots, n_{l-1}, n_{l} ; m_{1}, \ldots, m_{l-1}, 1 ; \tau, s\right) \\
\quad & =\left(\prod_{j=1}^{l} \frac{1}{n_{j}}\right) \frac{1}{n_{l}+\tau+s+1}+\frac{1}{n_{l}+\tau+s+1} Z\left(n_{1}, \ldots, n_{l-1} ; m_{1}, \ldots, m_{l-1} ; \tau, s\right) .
\end{aligned}
$$

Then, by induction on $m_{l}$ :

$$
\begin{aligned}
& Z\left(n_{1}, \ldots, n_{l-1}, n_{l} ; m_{1}, \ldots, m_{l-1}, m_{l} ; \tau, s\right) \\
& \quad=\frac{1}{n_{l}+(\tau+s+1) m_{l}}\left(m_{l} \prod_{j=1}^{l} \frac{1}{n_{j}}+Z\left(n_{1}, \ldots, n_{l-1} ; m_{1}, \ldots, m_{l-1} ; \tau, s\right)\right) .
\end{aligned}
$$

And by induction on $l$, if $u:=\tau+s+1$ :

$$
\begin{aligned}
& Z\left(n_{1}, \ldots, n_{l} ; m_{1}, \ldots, m_{l-1}, m_{l} ; \tau, s\right) \\
& \quad=\sum_{k=2}^{l} m_{k}\left(\prod_{j=1}^{k} \frac{1}{n_{j}}\right)\left(\prod_{j=k}^{l} \frac{1}{n_{j}+m_{j} u}\right)+\left(\prod_{j=2}^{l} \frac{1}{n_{j}+m_{j} u}\right) Z\left(n_{1} ; m_{1} ; \tau, s\right) .
\end{aligned}
$$

Formula 1.8. - Some computations show that

$$
Z\left(n_{1} ; m_{1} ; \tau, s\right)=\frac{1}{n_{1}+m_{1} u}\left(\frac{m_{1}}{n_{1}}+\frac{1}{s+1}+\frac{1}{\tau+1}-1\right) .
$$

Formula 1.9. - Combining the last formula we obtain

$$
\begin{aligned}
& Z\left(n_{1}, \ldots, n_{l} ; m_{1}, \ldots, m_{l} ; \tau, s\right) \\
& \quad=\frac{1}{u} \prod_{j=1}^{l} \frac{1}{n_{j}}+(u+1)\left(\frac{1}{(\tau+1)(s+1)}-\frac{1}{u}\right) \prod_{j=1}^{l} \frac{1}{n_{j}+m_{j} u} .
\end{aligned}
$$


Applied to the case of SIH singularities, i.e., $\tau=n+d s, t:=(n+1)+(d+1) s, m_{i}=N_{i}$ and $n_{i}=\nu_{i}$, one gets that $Z\left(\nu_{1}, \ldots, \nu_{l} ; N_{1}, \ldots, N_{l} ; n+d s, s\right)$ equals

$$
\frac{1}{t} \prod_{j=1}^{l} \frac{1}{\nu_{j}}+(t+1)\left(\frac{1}{(t-s)(s+1)}-\frac{1}{t}\right) \prod_{j=1}^{l} \frac{1}{\nu_{j}+N_{j} t}
$$

Remark 1.10. - In [7], J. Denef and F. Loeser, using $p$-adic integration and the GrothendieckLefschetz trace formula, showed that the local Denef-Loeser zeta function of the non-vanishing function germ $g^{P}$ verifies the equality

$$
Z_{\mathrm{top}, 0}\left(g^{P}, 0\right)=\sum_{J \subset\{0,1, \ldots, r\}} \chi_{J} \prod_{j \in J} \frac{1}{\nu_{i}}=1 .
$$

THEOREM 1.11. - If $P \in \operatorname{Sing}\left(C_{d}\right)$ then

$$
Z_{\mathrm{top}, 0}\left(\left(z-g^{P}(\bar{x})\right) z^{d}, \omega, s\right)=\frac{1}{t}+(t+1)\left(\frac{1}{(t-s)(s+1)}-\frac{1}{t}\right) Z_{\mathrm{top}, 0}\left(g^{P}, t\right) .
$$

COROLlary 1.12. - Let $f:=f_{d}+f_{d+1}+\cdots \in \mathbb{C}\left\{y_{0}, y_{1}, \ldots, y_{n}\right\}$ define a SIH singularity $(V, 0) \subset\left(\mathbb{C}^{n+1}, 0\right)$. Then its local Denef-Loeser zeta function satisfies the following equality

$$
\begin{aligned}
Z_{\mathrm{top}, 0}(V, s)= & \frac{\chi\left(\mathbb{P}^{n} \backslash C_{d}\right)}{t-s}+\frac{\chi\left(\check{C}_{d}\right)}{(t-s)(s+1)} \\
& +\sum_{P \in \operatorname{Sing}\left(C_{d}\right)}\left(\frac{1}{t}+(t+1)\left(\frac{1}{(t-s)(s+1)}-\frac{1}{t}\right) Z_{\mathrm{top}, P}\left(g^{P}, t\right)\right),
\end{aligned}
$$

where $g^{P}$ is a local equation of $C_{d}$ at $P$ and $t:=n+1+(d+1)$ s.

\section{The pole $s_{0}=-3 / d$ for $n=2$}

From now on, we will consider the case of surface singularities, i.e. $n=2$. Let

$$
f:=f_{d}+f_{d+1}+\cdots \in \mathbb{C}\left\{y_{0}, y_{1}, y_{2}\right\}
$$

be an analytic function such that its zero locus $(V, 0) \subset\left(\mathbb{C}^{3}, 0\right)$ defines a SIS singularity.

The germ $(V, 0) \subset\left(\mathbb{C}^{3}, 0\right)$ is an isolated surface singularity. Hence $H_{0}(F, \mathbb{C})$ and $H_{2}(F, \mathbb{C})$ are the only non-vanishing homology vector spaces on which the monodromy acts (we denote the Minor fiber by $F$ ). The only eigenvalue of the action of the monodromy on $H_{0}(F, \mathbb{C})$ is equal to 1 . The characteristic polynomial of the action of the complex monodromy on $H_{2}(F, \mathbb{C})$ is given by the formula

$$
\Delta_{V}(t)=\frac{\left(t^{d}-1\right)^{\chi\left(\mathbb{P}^{2} \backslash C_{d}\right)}}{(t-1)} \prod_{P \in \operatorname{Sing}\left(C_{d}\right)} \Delta^{P}\left(t^{d+1}\right),
$$

where $\Delta^{P}(t)$ is the characteristic polynomial (or Alexander polynomial) of the action of the complex monodromy of the germ $\left(C_{d}, P\right)$ on $H_{1}\left(F_{g^{P}}, \mathbb{C}\right)\left(F_{g^{P}}\right.$ denotes the corresponding Milnor fiber), e.g. see [2]. 
Proposition 2.1. - Let $(V, 0) \subset\left(\mathbb{C}^{3}, 0\right)$ a SIS singularity with tangent cone $C_{d} \subset \mathbb{P}^{2}$. Then:

(i) The poles of $Z_{\mathrm{top}, 0}(V, s)$ are contained in the set $\{-1,-3 / d\} \cup\left\{-\frac{\nu+3 N}{(d+1) N}\right\}$ whenever $-\nu / N$ is a pole of the local Denef-Loeser zeta function of the germ of $C_{d}$ at some point $P \in \operatorname{Sing}\left(C_{d}\right)$.

(ii) If $s_{0} \neq-3 / d$ is a pole of $Z_{\mathrm{top}, 0}(V, s)$ then $\exp \left(2 \mathrm{i} \pi s_{0}\right)$ is an eigenvalue of the monodromy zeta function of $V$.

(iii) Let $s_{0}=-3 / d$. If one of the following conditions holds then $\exp \left(2 \mathrm{i} \pi s_{0}\right)$ is an eigenvalue of the monodromy zeta function of $V$ :

- $\chi\left(\mathbb{P}^{2} \backslash C_{d}\right)>0$;

- $s_{0}$ is a pole of $Z_{\mathrm{top}, P}\left(C_{d}, s\right)$ at some point $P \in \operatorname{Sing}\left(C_{d}\right)$ and $\chi\left(\mathbb{P}^{2} \backslash C_{d}\right)=0$.

(iv) If $s_{0}=-3 / d$ is a multiple pole of $Z_{\mathrm{top}, 0}(V, s)$ then $\exp \left(2 \mathrm{i} \pi s_{0}\right)$ is an eigenvalue of the local monodromy zeta function at some singular point of $C_{d}$.

(v) If $s_{0}=-3 / d$ is not a pole of $Z_{\mathrm{top}, P}\left(C_{d}, s\right)$ for any $P \in \operatorname{Sing}\left(C_{d}\right)$, the residue of $Z_{\mathrm{top}, 0}(V, s)$ at $-3 /$ d equals $d \rho\left(C_{d}\right)$ where

$$
\rho\left(C_{d}\right):=\chi\left(\mathbb{P}^{2} \backslash C_{d}\right)+\chi\left(\check{C}_{d}\right) \frac{d}{d-3}+\sum_{P \in \operatorname{Sing}\left(C_{d}\right)} Z_{\mathrm{top}, P}\left(C_{d},-\frac{3}{d}\right) .
$$

Proof. - The formula in 1.12 for the local Denef-Loeser zeta function can be rewritten in the form

$$
Z_{\mathrm{top}, 0}(V, s)=\frac{3+\chi\left(\mathbb{P}^{2} \backslash C_{d}\right) s}{(3+d s)(s+1)}-\frac{s(2+s d)}{(1+s)(3+s d) t} \sum_{P \in \operatorname{Sing}\left(C_{d}\right)}\left((t+1) Z_{\mathrm{top}, P}\left(C_{d}, t\right)-1\right),
$$

where $t=3+(d+1) s$ and $Z_{\text {top }, P}\left(C_{d}, s\right)$ means the local Denef-Loeser zeta function of the germ at the point $P \in \operatorname{Sing}\left(C_{d}\right)$.

Recall that $Z_{\mathrm{top}, P}\left(C_{d}, 0\right)=1-$ see Remark 1.10 - for $P \in \operatorname{Sing}\left(C_{d}\right)$. Then, $s=0$ is not a pole of $\frac{1}{s}\left((s+1) Z_{\mathrm{top}, P}\left(C_{d}, s\right)-1\right)$ and $s=-\frac{3}{d+1}$ is not a pole of $Z_{\mathrm{top}, 0}(V, s)$ if different from -1 (that is, $d \neq 2$ ). The other candidate poles are evident and this argument proves (i).

The above formula of the characteristic polynomial $\Delta_{V}(t)$ of the complex monodromy also gives (iii).

For (ii), we must verify the statement for $s_{0}=-\frac{\nu+3 N}{(d+1) N}$ where $-\nu / N$ is a pole of the local Denef-Loeser zeta function of the germ of $C_{d}$ at some point $P \in \operatorname{Sing}\left(C_{d}\right)$. Let $\varepsilon_{1}:=\exp \left(-2 \mathrm{i} \pi \frac{\nu}{N}\right)$. According to the monodromy conjecture for curves [21], $\varepsilon_{1}$ is a root of $\Delta^{P}(t)$ and by the previous formula, $\varepsilon:=\exp \left(2 \mathrm{i} \pi s_{0}\right)$ is a root of $\Delta^{P}\left(t^{d+1}\right)$, since $\varepsilon^{d+1}=\varepsilon_{1}$.

Hence, for the cases $\chi\left(\mathbb{P}^{2} \backslash C_{d}\right) \geqslant 0$, or $\chi\left(\mathbb{P}^{2} \backslash C_{d}\right)<0$ where $\varepsilon$ is not a root of $\left(t^{d}-1\right)$, we are done.

For the rest of the cases, we will prove that $s_{0}=-3 / d$. Since $\varepsilon^{d}=1$ then $s_{0} d \in \mathbb{Z}^{*}$, i.e. there exists $k>0$ such that $(\nu+3 N) d=k(d+1) N$. It implies that $\nu d=N(d(k-3)+k)$ or equivalently $\nu / N=k-3+k / d$. In the case of plane curves one has $0<\nu / N \leqslant 1$ which implies that $0<k-3+k / d \leqslant 1$, i.e. $3 \frac{d}{d+1}<k \leqslant 4 \frac{d}{d+1}<4$.

Disregarding curves of degree $d=1$, we will assume $d \geqslant 2$ and hence $2<k<4$, i.e. $k=3$. Note that if $k=3$ then $-\nu / N=-3 / d$ and $s_{0}=-3 / d$. We have proved (ii).

The remaining statements are direct consequences of the monodromy conjecture for curves and the formulæ for the Denef-Loeser zeta functions and for the characteristic polynomial of the monodromy.

Example 2.2. - We consider the case $d \leqslant 3$ and $\chi\left(\mathbb{P}^{2} \backslash C_{d}\right) \leqslant 0$. It is easily seen that only four cases are possible: (I) $C_{d}$ has two lines $L_{1}$ and $L_{2}$; (II) $C_{d}$ is the union of three lines meeting at 
Table 1

\begin{tabular}{rcc}
\hline & $Z_{\text {top }, 0}(V, s)$ & $\Delta_{V}(t)$ \\
\hline (I) & $\frac{4+s}{(1+s)(4+3 s)}$ & $\frac{t^{3}-1}{t-1}$ \\
(II) & $\frac{11}{(1+s)(11+12 s)}$ & $\frac{\left(t^{12}-1\right)\left(t^{4}-1\right)}{\left(t^{3}-1\right)(t-1)}$ \\
(III) & $\frac{3 s^{2}+6 s+4}{4(1+s)^{3}}$ & $\frac{\left(t^{4}-1\right)^{3}}{(t-1)}$ \\
(IV) & $\frac{3 s+15}{(1+s)(15+16 s)}$ & $\frac{\left(t^{16}-1\right)\left(t^{4}-1\right)}{\left(t^{8}-1\right)(t-1)}$ \\
\hline
\end{tabular}

one point; (III) $C_{d}$ is the union of three generic lines and (IV) $C_{d}$ is the union of a smooth conic and a tangent line. The computations for both invariants are in Table 1.

With these examples the monodromy conjecture for any SIS singularity of multiplicity $d=2$ or 3 is proved. From now on we will assume that $d>3$.

We have proved the monodromy conjecture for SIS in all but two cases:

$(N-1) \chi\left(\mathbb{P}^{2} \backslash C_{d}\right)=0, s_{0}=-3 / d$ is not a pole for the local Denef-Loeser zeta function at any singular point in $C_{d}$ and $\rho\left(C_{d}\right) \neq 0$.

$(N-2) \chi\left(\mathbb{P}^{2} \backslash C_{d}\right)<0$.

Our goal in this section is to determine which curves $C_{d}$ can occur in the cases $(N-1)$ and $(N-2)$. The condition about the Euler-Poincare characteristic implies that $C_{d}$ has at least two irreducible components and all of them are rational curves - see [16]. Moreover, Veys' theorem shows that the irreducible components of $C_{d}$ are components of curves in a pencil $\Lambda$ in $\mathbb{P}^{2}$ (defined by a rational function $R$ ) of rational curves. If $B$ is the set of base points of $\Lambda$ then the generic fiber of $R$ is a rational curve with at most two punctures. These pencils have been studied by H. Kashiwara in [17] and T. Kizuka [18] - see Appendix A.

In the case $(N-2), C_{d}$ has at least three irreducible components and we can apply Corollary 3.6 in [32] and show that after Veys' process we are in a configuration of type (A) in Veys' theorem.

In order to study $(N-1)$ we introduce the notion of a bad divisor.

Definition 2.3. - We say that a degree $d$ effective divisor $D$ on $\mathbb{P}^{2}(d>3)$ is a bad divisor if $\chi\left(\mathbb{P}^{2} \backslash D\right) \leqslant 0$ and $s_{0}=-3 / d$ is not a pole of $Z_{\mathrm{top}, P}\left(g_{D}^{P}, s\right)$, for any singular point $P$ in its support $D_{\text {red }}$, where $g_{D}^{P}$ is the local equation of the divisor $D$ at $P$.

Note that in order to deal with the case $(N-1)$ it would be enough to define a bad divisor as a divisor verifying $\chi\left(\mathbb{P}^{2} \backslash D\right)=0$ and the same condition for the pole $s_{0}=-3 / d$. We need this more general definition to prove Theorem 2.15.

The residue $\rho\left(C_{d}\right)$ is related to the Denef-Loeser zeta function associated with any effective divisor $D$ on $\mathbb{P}^{2}$ introduced by $\mathrm{W}$. Veys. Let $D$ be an effective divisor on $\mathbb{P}^{2}$ of degree $d(d>3)$. Let $D=a_{1} D_{1}+\cdots+a_{r} D_{r}$ and set $\check{D}_{i}:=D_{i} \backslash \operatorname{Sing}\left(D_{\text {red }}\right)$. From Section 1, the Denef-Loeser zeta function of the divisor $D$ on $\mathbb{P}^{2}$ can be rewritten as follows

$$
z(D, s)=\chi\left(\mathbb{P}^{2} \backslash D\right)+\sum_{i=1}^{r} \frac{\chi\left(\check{D}_{i}\right)}{1+a_{i} s}+\sum_{P \in \operatorname{Sing}\left(D_{\mathrm{red}}\right)} Z_{\mathrm{top}, P}(D, s) .
$$

In the SIS case, the curve $C_{d}$ is reduced, i.e. $a_{i}=1$, and then $\rho\left(C_{d}\right)$ is equal to the value $z\left(C_{d},-3 / d\right)$. In general, for a divisor $D$ in $\mathbb{P}^{2}$ we define $\rho(D):=z(D,-3 / d) \in \mathbb{Q} \cup\{\infty\}$. We are also going to use another interpretation of the rational number (or infinity) $\rho(D)$. 
Let $D$ be an effective divisor on $\mathbb{P}^{2}$ of degree $d>3$, then $-\frac{3}{d} D$ is a $\mathbb{Q}$-canonical divisor on $\mathbb{P}^{2}$. Let $\pi: X \rightarrow \mathbb{P}^{2}$ be the minimal embedded resolution for the support of $D$ in $\mathbb{P}^{2}$. The map $\pi$ is a sequence of blowing-ups centered at infinitely near points of points in $\operatorname{Sing}\left(D_{\text {red }}\right)$ such that the divisor $\pi^{*} D$ is a normal crossing divisor. Let $K_{X}$ be the $\mathbb{Q}$-canonical divisor on the surface $X$ obtained from the pull-back of $-\frac{3}{d} D$ - see Remark 2.4. The irreducible components of $K_{X}$ are the strict transforms of the irreducible components of $D$ and the exceptional components over each singular point of $D_{\text {red }}$. The corresponding multiplicities in $K_{X}$ are:

- $-3 \frac{a_{i}}{d}$ for the strict transform of the irreducible component $D_{i}$ of $D$.

- $\frac{-3}{d} N_{i}+\nu_{i}-1$, for any exceptional component $E_{i}$, associated with a point $P \in \operatorname{Sing}\left(D_{\text {red }}\right)$, where $N_{i}$ and $\nu_{i}$ are defined as in Section 1.

Remark 2.4. - For instance, if $\pi_{1}: Y \rightarrow \mathbb{P}^{2}$ is the blow-up at some point $P$ then $K_{Y}=\pi^{*}\left(-\frac{3}{d} D\right)+E$. In general, the map $\pi: X \rightarrow \mathbb{P}^{2}$ is a composition of blow-ups. By canonical pull-back of a $\mathbb{Q}$-divisor $K$ we mean that if $K$ is the divisor on $\mathbb{P}^{2}$ of a multivaluated meromorphic 2-form $\omega$, then its canonical pull-back $K_{X}$ is the divisor of the pull-back $\pi^{*} \omega$ of $\omega$.

\section{1. $\zeta$-invariants of $\mathbb{Q}$-canonical divisors}

In this subsection, $X$ is a rational surface and $K_{X}:=\sum_{i=1}^{r}\left(\nu_{i}-1\right) E_{i}$ is a $\mathbb{Q}$-canonical divisor on $X$ with normal crossings. Let $G$ be the dual graph of $K_{X}$ with vertices $V(G)$ and edges $E(G)$. The weight of the vertex $v_{i}$ associated with $E_{i}$ is defined by $w_{v_{i}}:=\nu_{i}$; for an edge $e$ we denote by $V(e)$ the set of its endpoints (or extremities) and we set $w_{e}:=1$.

This graph can also be weighted by the self-intersection numbers $a_{i}:=E_{i}^{2}$ of the irreducible components $E_{i}$ of $K_{X}$ on the surface $X$. A subgraph $G_{1}$ of $G$ is a graph such that $V\left(G_{1}\right) \subset V(G)$ and any edge in $G$, with extremities in $V\left(G_{1}\right)$, is an edge in $G_{1}$.

Definition 2.5. - We say that a subgraph $G_{1}$ of $G$ is a set of bamboos if any connected component of the graph $G_{1}$ is linear, the irreducible components of $K_{X}$ associated with the vertices of $G_{1}$ are rational curves and if $v \in V(G)$ is an endpoint of $G_{1}$, then its valency in $G$ is less than 3.

In such a case, each connected component of $G_{1}$ is called a bamboo. A bamboo is of type 1 (resp. 2) if it has one (resp. two) neighboring vertex (resp. vertices) in $G$.

Let $V(B):=\left\{v_{i_{1}}, \ldots, v_{i_{r}}\right\}$ be the set of vertices of a bamboo $B$ of $G$. The intersection matrix of $B$ is the integer matrix $A=\left(a_{i j}\right) \in M(r, \mathbb{Z})$ such that if $j \neq k$, then $a_{j k}$ is the number of edges between $v_{i_{j}}$ and $v_{i_{k}}$, i.e. the intersection number between $E_{i_{j}}$ and $E_{i_{k}}$, and $a_{j j}:=a_{j}$. The determinant of the bamboo is $\operatorname{det}(B):=\operatorname{det}(-A)$ (which does not depend on the order of the vertices of $B$, e.g. see [31]).

Definition 2.6. - Let $G_{1} \neq G$ be a set of bamboos of $G$. We define the graph $G / G_{1}$ which has weighted vertices, weighted edges and weighted arrows as follows:

- The set of vertices $V\left(G / G_{1}\right)$ is nothing but $V(G) \backslash V\left(G_{1}\right)$ and they are weighted as in $G$.

- The set of edges $E\left(G / G_{1}\right)$ has two types of elements. Edges of $G$ not intersecting $G_{1}$ produce edges of $G / G_{1}$; theses edges are weighted by 1 . Each bamboo of type 2 produces also one edge with the obvious extremities and weighted by the determinant of the bamboo.

- The set $A\left(G / G_{1}\right)$ of arrows of the graph $G / G_{1}$ is in one-to-one correspondence with the set of bamboos of $G_{1}$ of type 1 . It is weighted by the determinant of the corresponding bamboo. Note that each arrow $a$ in $G / G_{1}$ has only one neighbor vertex $v_{a}$.

DEFINITION 2.7. - Let $K_{0}$ be the reduced subdivisor of $K_{X}$ consisting of all the irreducible components $E_{i}$ of $K_{X}$ such that $\nu_{i}=0$. Let $G_{0}$ be the dual graph of $K_{0}$. We say that $K_{X}$ is 
admissible if $G_{0}$ is a set of bamboos. In such a case we define

$$
\zeta_{K_{X}}:=\chi(\check{X})+\sum_{v \in V\left(G / G_{0}\right)} \frac{\chi\left(\check{E}_{v}\right)}{w_{v}}+\sum_{e \in E\left(G / G_{0}\right)} w_{e} \prod_{v \in V(e)} \frac{1}{w_{v}}+\sum_{a \in A\left(G / G_{0}\right)} \frac{w_{a}}{w_{v_{a}}} \in \mathbb{Q} .
$$

Note that the invariant $\zeta_{K_{X}}$ does not change if we add irreducible components which are not in the support of $K_{X}$. The adjunction formula and a standard induction argument are the key points of the following result, which is a generalization of one of Veys' results in [31, Theorem 3.3].

Proposition 2.8. - Let $G_{1}$ be a set of bamboos such that for any $v \in V(G) \backslash V\left(G_{1}\right)$ we have $w_{v} \neq 0$. Then:

$$
\zeta_{K_{X}}=\chi(\check{X})+\sum_{v \in V\left(G / G_{1}\right)} \frac{\chi\left(\check{E}_{v}\right)}{w_{v}}+\sum_{e \in E\left(G / G_{1}\right)} w_{e} \prod_{v \in V(e)} \frac{1}{w_{v}}+\sum_{a \in A\left(G / G_{1}\right)} \frac{w_{a}}{w_{v_{a}}} \in \mathbb{Q} .
$$

PROPERTY 2.9. - If $v \in V(G)$ is a vertex with weight zero then the adjunction formula implies that the sum of the neighbor weights is equal to the valency of the vertex minus 2 . It turns out that each connected component of $K_{0}$ consists only of one rational curve $E$. This implies that:

- if the bamboo is of type 2 and gives an edge $e \in E\left(G / G_{0}\right)$, then the weights of the vertices in $V(e)$ are opposite to each other,

- and if it is of type 1 and gives an arrow $a \in A\left(G / G_{0}\right)$, then its weight in $V(a)$ is equal to -1 .

We examine the behavior of these invariants under blow-ups.

Proposition 2.10. - Let $X$ be a rational surface and let $K_{X}$ be an admissible $\mathbb{Q}$-canonical divisor. Let $\pi: Y \rightarrow X$ be the blowing-up of a point $P \in X$ and let $K_{Y}$ be the canonical pull-back of $K_{X}$. Then, $K_{Y}$ is admissible and:

(i) If $P$ does not belong to $K_{0} \backslash \operatorname{Sing}\left(K_{X \text {,red }}\right)$, then $\zeta_{K_{X}}=\zeta_{K_{Y}}$.

(ii) If $P$ belongs to $K_{0} \backslash \operatorname{Sing}\left(K_{X, \mathrm{red}}\right)$, let $B_{1}$ be the bamboo of $K_{0}$ containing $P$. Let us suppose that the self-intersection of $B_{1}$ is $-a$ and the neighbors of $B_{1}$ in $G$ have weights $w$ and $-w$ (or $w=-1$ if it is of type 1$)$. Then, the corresponding bamboo $B_{2}$ in $K_{Y}$ has self-intersection $-a-1$ and

$$
\zeta_{K_{X}}+1-\frac{1}{w^{2}}=\zeta_{K_{Y}}
$$

Proof. - Let $E_{P}$ be the exceptional divisor of the blowing-up $\pi: Y \rightarrow X$ of $X$ at the point $P \in X$. It is easily seen that if $K_{X}$ is admissible, this is also the case for $K_{Y}$. We restrict ourselves to the proof of the most relevant cases.

The proof of the remaining cases is based on the study of the contribution of the point $P$ (and its neighbors) to $\zeta_{K_{X}}$ and the exceptional curve $E_{P}$ (and its neighbors) to $\zeta_{K_{Y}}$. What we mean by contribution is that there exists $\eta \in \mathbb{Q}$ such that $\zeta_{K_{X}}=\eta+\left(\right.$ contribution to $\left.\zeta_{K_{X}}\right)$ and $\zeta_{K_{Y}}=\eta+$ (contribution to $\left.\zeta_{K_{Y}}\right)$.

CASE 1. - The point $P$ is a double point of $K_{X, \mathrm{red}}, P \in E_{i} \cap E_{j}, \nu_{i}=0$.

Let us consider again weights $w_{i}(=0), w_{j}$. The curve $E_{i}$ gives a bamboo in $K_{X}$, with determinant $a$ and neighbor weights $-w_{j}$ and $w_{j}$. The contribution of this bamboo to $\zeta_{K_{X}}$ is 
$-a / w_{j}^{2}$. The strict transform of $E_{i}$ in $K_{Y}$ is a bamboo of determinant $a+1$ and neighbor weights $-w_{j}, w_{j}$. The intersection point between the new exceptional divisor $E_{P}$ and $E_{j}$ contributes with $1 / w_{j}^{2}$. Hence its contribution to $\zeta_{K_{Y}}$ is:

$$
-\frac{a+1}{w_{j}^{2}}+\frac{1}{w_{j}^{2}} .
$$

CASE 2. - The point $P$ is smooth in $K_{X \text {,red }} P \in \check{E}_{i}$ and $\nu_{i}=0$.

Let us assume the notation of (ii). The contribution of the bamboo $E_{i}$ to $\zeta_{K_{X}}$ is equal to $-a / w^{2}$. The strict transform of $E_{i}$ is also a bamboo in $K_{Y}$ whose contribution is $\frac{-a-1}{w^{2}}$. But in this case the exceptional divisor $E_{P}$ has $\nu_{E_{P}}=1$, so it is not in the support of $K_{Y}$ and the Euler-Poincaré characteristic of the complement of $K_{Y}$ in $Y$ differs by 1 from the one of $K_{X}$ in $X$.

COROLlARY 2.11. - Let $X$ be a rational surface and let $K_{X}$ be an admissible $\mathbb{Q}$-canonical divisor. Let $\pi: Y \rightarrow X$ be the blowing-up at a point $P \in X$ and let $K_{Y}$ be the canonical pull-back of $K_{X}$. If $P$ belongs to $K_{0} \backslash \operatorname{Sing}\left(K_{X \text {,red }}\right)$ then

$$
\chi\left(X \backslash K_{X}\right)<\chi\left(Y \backslash K_{Y}\right) .
$$

In particular, there is a unique blowing-up process in which the following holds: $\zeta_{K_{X}} \neq \zeta_{K_{Y}}$ and $\chi\left(X \backslash K_{X}\right)<\chi\left(Y \backslash K_{Y}\right)$. Such a process is the blowing-up of $X$ at $P \in K_{0} \backslash \operatorname{Sing}\left(K_{X \text {,red }}\right)$ having valency 2 and whose neighbor vertices have weights $\pm w, w \neq 1$.

The following result computes the invariant $\zeta_{K_{X}}$ in the hypothesis of Veys' theorem.

Proposition 2.12. - Let $K_{X}$ be an admissible $\mathbb{Q}$-canonical divisor on a ruled rational surface $X$ such that its support is of type (A) or (B). Then $\zeta_{K_{X}}=0$.

Proof. - Using Nagata transformations which do not change $\zeta_{K_{X}}$ we can assume $\Sigma=\mathbb{P}^{1} \times \mathbb{P}^{1}$.

Let us consider $S$ and $F$ the general 0 -section and the general fiber respectively. It is known that canonical divisors are linearly equivalent to $-2 S-2 F$.

For case (A), let us denote by $S_{0}$ the section and by $F_{1}, \ldots, F_{r}$ the fibers. One can assume $r \geqslant 2$. Let $K_{\Sigma}$ be an admissible canonical divisor with support contained in the curves above. Then $K_{\Sigma}=-2 S_{0}+\sum_{j=1}^{r} k_{i} F_{i}$, with $\sum_{j=1}^{r} k_{i}=-2$. The result follows by applying the formula in the definition of the invariant $\zeta_{K_{\Sigma}}$.

For case (B), let us denote by $S_{0}, S_{1}$ the sections and by $F_{1}, \ldots, F_{r}$ the fibers. One can assume $r \geqslant 2$. Let $K_{\Sigma}$ be an admissible canonical divisor with support contained in the curves above. Since $\Sigma=\mathbb{P}^{1} \times \mathbb{P}^{1}$ we can interchange fibers and sections if necessary.

In order for $K_{\Sigma}$ to be admissible, we can suppose that $K_{\Sigma}=a_{0} S_{0}+a_{1} S_{1}+\sum_{j=1}^{r} k_{i} F_{i}$, with $\sum_{j=1}^{r} k_{i}=-2, a_{0}+a_{1}=-2$ and $a_{0}, a_{1} \neq-1$. Once again, the result is a consequence of the definition of the invariant $\zeta_{K_{\Sigma}}$.

\section{2. $\zeta$-invariant and bad divisors}

Next we will relate $\rho\left(C_{d}\right)$ with the invariants defined above.

Lemma 2.13. - Let $D$ be an effective divisor on $\mathbb{P}^{2}$ of degree $d, d>3$. Let $\pi: X \rightarrow \mathbb{P}^{2}$ be the minimal resolution of $\operatorname{Sing}\left(D_{\text {red }}\right)$. Let us suppose that, for any singular point $P \in D_{\text {red }}$, the local Denef-Loeser zeta function of $D$ at $P$ does not have a pole at $-3 / d$. Let $K_{X}$ be the canonical pull-back of $-\frac{3}{d} D$ by $\pi$. Then $K_{X}$ is admissible and $\rho(D)=\zeta_{K_{X}}$. 
Proof. - Let $E$ be an exceptional component of the embedded resolution of $D$ at a point $P$ and let $N$ be the multiplicity of $E$ in the total transform of $D$ and let $\nu-1$ be the multiplicity in the pull-back of a local generator of the sheaf of 2-holomorphic forms.

It is easily seen that the weight of $E$ in $K_{X}$ equals $-(3 / d) N+\nu$. If $-3 / d$ is not a pole for the local Denef-Loeser zeta function of $D$ at $P$ then from Veys' results on the monodromy conjecture for curves [30], we can assume that the valency of $E$ in the divisor is either 1 or 2 . This fact implies that $K_{X}$ is admissible.

Example 2.14. - Let $D \subset \mathbb{P}^{2}$ be the union of two smooth conics $C_{1}$ and $C_{2}$ which meet at only one point $\{P\}=C_{1} \cap C_{2}$. Consider $D$ as divisor of degree 4 and $K:=(-3 / 4) D$ as a $\mathbb{Q}$-rational divisor on $\mathbb{P}^{2}$. Let $\pi: X \rightarrow \mathbb{P}^{2}$ be the minimal embedded resolution of the singularity of $D$ at the point $P$. The rational surface $X$ has the configuration of curves and the corresponding associated invariants shown in Fig. 1.

The dual graph of the resolution $G$ has only one bamboo $G_{0}$ and the corresponding graph $G / G_{0}$ is shown in Fig. 2.

The $\zeta_{K_{X}}$-invariant is non-zero because

$$
\zeta_{K_{X}}=\frac{1}{\frac{1}{2}}+(-1)(-1)+2 \frac{1}{\frac{1}{4}}+2 \frac{1}{\frac{-1}{2} \frac{1}{2}}+\frac{1}{\frac{-1}{2}(-1)}+2 \frac{1}{\frac{1}{4}(-1)} \neq 0 .
$$

Thus the $\zeta_{K_{X}}$-invariant does not "behave well" under blow-ups and we only have partial control over it.

Below we compute $Z_{\text {top }, 0}\left(V_{D}, s\right)$ and $\Delta_{V}(t)$ for a SIS singularity $\left(V_{D}, 0\right) \subset\left(\mathbb{C}^{3}, 0\right)$ whose tangent cone is $D$. In this case $\chi\left(\mathbb{P}^{2} \backslash D\right)=0$, and $s_{0}=-3 / 4$ is not a pole of $Z_{\mathrm{top}, P}(D, s)$ for the germ of curve $D$ at $P$. Hence $D$ is a bad divisor on $\mathbb{P}^{2}$. Since the residue $\rho(D)=\zeta_{K_{X}} \neq 0$, then $s_{0}=-3 / 4$ is a simple pole of $Z_{\text {top }, 0}\left(V_{D}, s\right)$ and, as one can easily check, $\exp \left(-2 \mathrm{i} \pi \frac{3}{4}\right)$ is a root of $\Delta_{V}(t)$.

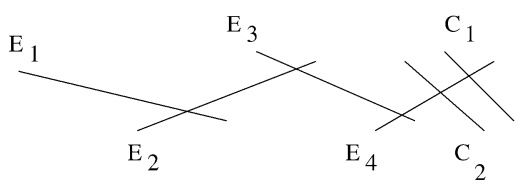

\begin{tabular}{|c|c|c|c|}
\hline & $\left(N_{i}, \nu_{i}\right)$ & $w_{i}$ & $E_{i}^{2}$ \\
\hline$E_{1}$ & $(2,2)$ & $\frac{1}{2}$ & -2 \\
\hline$E_{2}$ & $(4,3)$ & 0 & -2 \\
\hline$E_{3}$ & $(6,4)$ & $-\frac{1}{2}$ & -2 \\
\hline$E_{4}$ & $(8,5)$ & -1 & -1 \\
\hline$C_{1}, C_{2}$ & $(1,1)$ & $\frac{1}{4}$ & 0 \\
\hline
\end{tabular}

Fig. 1.

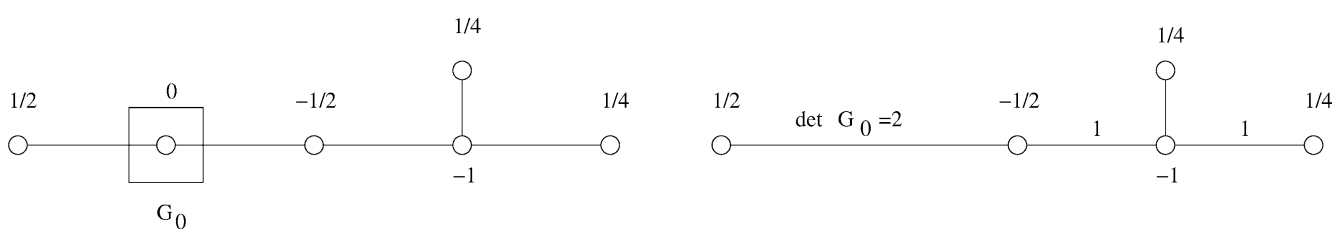

Fig. 2. 


$$
\begin{aligned}
& Z_{\mathrm{top}, P}(D, s)=\frac{3 s+5}{(1+s)(5+8 s)} \\
& Z_{\mathrm{top}, 0}\left(V_{D}, s\right)=\frac{130 s+20 s^{2}+87}{(1+s)(3+4 s)(29+40 s)} \\
& \Delta_{V}(t)=\frac{\left(t^{5}-1\right)\left(t^{40}-1\right)}{\left(t^{10}-1\right)(t-1)}
\end{aligned}
$$

We have mentioned that the Euler-Poincaré characteristic condition on a bad divisor $D$ implies that $D$ has at least two irreducible components, which happen to be rational curves. In the definition of a bad divisor we imposed that $s_{0}=-3 / d$ is not a pole (although $s_{0}$ might be a candidate pole) of $Z_{\mathrm{top}, P}(D, s)$ for any singularity $P$ in $D_{\text {red }}$. A direct application of Veys' Theorem to a bad divisor $D$ allows for an extension of the curve $D$ to another curve $D^{\prime} \supset D$, having $\chi\left(\mathbb{P}^{2} \backslash D^{\prime}\right) \leqslant 0$, such that the following diagram holds:

$$
\Sigma \stackrel{\pi_{3}}{\longleftarrow} X_{2} \stackrel{\pi}{\longrightarrow} \mathbb{P}^{2}
$$

where $\pi$ is a composition of blowing-ups with center in $D^{\prime}$ and $\pi_{3}$ is a composition of blowingdowns with exceptional curve contained in $\pi^{-1}\left(D^{\prime}\right)$ and such that $\Sigma$ is a ruled surface. We call this a Veys' process. The configuration $T:=\pi_{3}\left(\pi^{-1}\left(D^{\prime}\right)\right)$ consists of one of the types (A) or (B) in Veys' theorem. In particular, the curve $D^{\prime}$ is a union of fibers and sections of a particular kind of rational pencils studied by H. Kashiwara in [17] and by T. Kizuka [18] - see Appendix A. Note that several pencils may match for a given curve $D \subset \mathbb{P}^{2}$ and thus several different constructions can be acquired. Let us call $R$ the rational function defining such a pencil $\Lambda$ and let us define $\bar{R}:=\pi^{*} R$.

Veys' processes can be decomposed in three stages by factoring $\pi$ as $\pi_{2} \circ \pi_{1}$.

- In the first step we consider the minimal embedded resolution $\pi_{1}: X_{1} \rightarrow \mathbb{P}^{2}$ of the local singularities of $D_{\text {red }} \subset \mathbb{P}^{2}$. At this stage, $\rho\left(C_{d}\right)$ is already computed as $\zeta_{K_{X_{1}}}$, where $K_{X_{1}}$ is the canonical pull-back of $(-3 / d) C_{d}$. Since $-3 / d$ is not a pole of $Z_{\mathrm{top}, P}\left(C_{d}, s\right)$ for any $P \in \operatorname{Sing}\left(C_{d}\right)$, then $K_{X_{1}}$ is admissible.

- Next, we consider the resolution $\pi_{2}: X_{2} \rightarrow X_{1}$ of the indeterminacy locus of the pencil $\Lambda^{*} \subset X_{1}$ defined by the function $\pi_{1}^{*} R$ - it might happen that $\pi_{2}$ is the identity map.

- Finally, let $\pi_{3}: X_{2} \rightarrow \Sigma$ be the contraction of $X_{2}$ onto a rational ruled surface $\Sigma$.

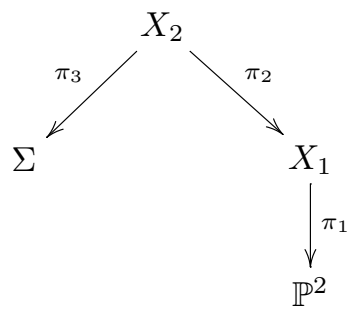

From now on, we can also assume that $\pi$ is minimal in the following sense - see [32, (4.3)]: The map $\pi: X_{2} \rightarrow \mathbb{P}^{2}$ is the minimal morphism that resolves the indeterminacies of the morphism induced by the pencil $\Lambda$.

The irreducible components of the strict transform of $D$ in $X_{2}$ have to be either irreducible components of members of the total transform of the pencil $\Lambda$ or sections of the map $\bar{R}: X_{2} \rightarrow \mathbb{P}^{1}$ (recall that a curve $E$ is a section if the restricted map $\bar{R} \mid: E \rightarrow \mathbb{P}^{1}$ is surjective). 
We have proved in the previous lemma that the residue $\rho(D)$ of a bad divisor $D$ is equal to the $\zeta_{K_{X_{1}}}$-invariant of any admissible $\mathbb{Q}$-canonical divisor on $X_{1}$. Proposition 2.12 shows that (in the final configurations) the $\zeta_{K_{\Sigma}}$-invariant is zero.

In the following theorem we describe bad divisors $D$ with $\rho(D) \neq 0$ - in particular, curves of type $(N-1)$ - in terms of Veys' theorem. Using Kashiwara's notation, we show that they come from a rational pencil $\Lambda$ on $\mathbb{P}^{2}$ of type $(0,1)$, i.e. a pencil of rational curves having only one base point and such that any fiber minus the base point is isomorphic to $\mathbb{C}$ - see Appendix A. We are looking for bad divisors having non-zero $\rho(D)$. For this purpose we must understand the behavior of the maps $\pi_{2}$ and $\pi_{3}$, and find out when it is possible that at least one of the blow-ups in $\pi_{2}$ or $\pi_{3}$ matches the hypothesis of 2.10 (ii), with $1 \neq w^{2}$.

THEOREM 2.15. - Let $D$ be a bad divisor on $\mathbb{P}^{2}$. If $\rho(D) \neq 0$, then $D$ can be transformed, by means of Veys' process, into a curve $T$ of type (A) with at least three fibers and such that the irreducible components of $D$ are in a pencil $\Lambda$ of type $(0,1)$.

Moreover, at least one generic fiber (resp. two) of $\Lambda$ is contained in $D$ if the pencil has two exceptional fibers (resp. one).

Proof. - Note that the first part of the theorem was already proved in Corollary 3.6 in [32] for the case where the Euler-Poincaré characteristic verifies $\chi\left(\mathbb{P}^{2}-D\right)<0$.

The irreducible components of $D$ may correspond either to components of the fibers of the pencil or to sections. Each base point of $\Lambda$ produces a section of the pencil in $X_{2}$. Therefore, if a component was transformed into a section after Veys' process, we would obtain a curve of type (B) (we will show that this is not possible).

Since the $\zeta$-invariant of the surfaces $X_{1}$ and $\Sigma$ are different, at least one of the blow-ups in $\pi_{2}: X_{2} \rightarrow X_{1}$ or $\pi_{3}: X_{2} \rightarrow \Sigma$ is as in 2.10(ii). This means that we have blown up at a point $P \in E$ which is in the conditions of Proposition 2.10 or Corollary 2.11. Let us denote by $E_{P}$ the exceptional divisor in the corresponding rational surface, which comes from a blowing-up at $P \in E$ changing the $\zeta$-invariant.

CASE 1. - The changes of the $\zeta$-invariant happen in $\pi_{3}: X_{2} \rightarrow \Sigma$.

Since the weight of $E_{P}$ in the canonical divisor is equal to 1, it will be either an exceptional component for $\pi=\pi_{2} \circ \pi_{1}: X_{2} \rightarrow \mathbb{P}^{2}$ or an irreducible component of $D^{\prime} \backslash D$ in Veys' theorem.

(i) In the first case, the divisor $E$ has more than 2 neighbors in the exceptional divisor of $\pi: X_{2} \rightarrow \mathbb{P}^{2}$. Its weight in the canonical divisor is 0 . Note that it is not possible for this component to become a component of valency 2 in the minimal resolution $\pi_{1}$. The reason is that, in this case, the neighbor component which is not blown-down has weight equal to -1 and thus the $\zeta_{K}$-invariant does not change. Therefore $E$ has more than 2 neighbors in the exceptional divisor of $\pi_{1}: X_{1} \rightarrow \mathbb{P}^{2}$, and $-3 / d$ is a pole of the local Denef-Loeser zeta function of at least one local singularity of $D_{\text {red }}$. This implies that $D$ is not a bad divisor.

(ii) In the second case, the Euler-Poincare characteristic of the complement changes. This implies that $D^{\prime}$ is transformed into a curve $T$ of type (A) with at least three fibers. As seen above, in this case the Euler-Poincaré characteristic of the complement is increased by one from the Euler-Poincaré characteristic on the curve of type (A) or (B). Since we must keep this invariant non-positive, in the curve of type (A) or (B) we must have a negative Euler-Poincaré characteristic. This is only possible if the curve is of type (A) with at least three fibers - see Corollary 2.11. In particular, in this case no component of $D$ is a section.

CASE 2. - The changes of the $\zeta$-invariant occur at some blow-up in $\pi_{2}: X_{2} \rightarrow X_{1}$. 
In this case the point $P$, at which the blow-up is performed, is a base point of the strict transform of the pencil $\Lambda$. The exceptional component $E$ which contains $P$ has weight 0 in the canonical divisor, valency 2 in the dual graph and neighbors with weights $\pm w(w \neq \pm 1)$.

Once again, the weight of $E_{P}$ is 1 . After several blowing-ups we get a tree $\mathcal{T}$ of exceptional curves based on $E$. Note that the component which intersects our original $E$ is weighted by 1 . In $\mathcal{T}$ there is at least one section which has weight greater than 0 . If $E$ is also a section, we have at least two sections and thus a pencil of type $(0,2)$. We have a curve of type (B). Using the kind of arguments shown in Proposition 2.12, the weights $s_{1}$ and $s_{2}$ of the two sections in the corresponding canonical divisor in $\Sigma$ verify $s_{1}+s_{2}=0$. In this case the weight of $E$ is 0 and the weight of the other section is greater than 0 , which is impossible. Hence, the divisor $E$ is not a section.

Therefore the curve $E$ is a component of a fiber of the pencil. This implies that either $E$ disappears in one of the contractions of $\pi_{3}$ or it remains in $\Sigma$. In any case, one of the three neighbors must be contracted. Due to the minimality of $\pi$ at least one of the exceptional components of the tree $\mathcal{T}$ has to remain on the surface $\Sigma$. Thus, one of the original neighbors is contracted. By the adjunction formula, this means that the other one has weight -1 , contradicting the hypothesis. Thus this case is also impossible.

Therefore, the only possible situation is given in Case 1(ii). The curve $E_{P}$ gives an irreducible component $\pi\left(E_{P}\right)$ of $D^{\prime} \backslash D$, which is a special fiber of the pencil $\Lambda$ since reduced members of of pencils of type $(0,1)$ are irreducible. Such pencils have at most two special fibers and then $D$ contains at least one generic fiber of $\Lambda$.

Remark 2.16. - The general ideas of the proof of Case 2 have been pointed out to us by $\mathrm{W}$. Veys to whom we are grateful. Our original proof was longer and it was based on the analysis of Kizuka's work [18].

Example 2.17. - As we have seen in the previous example, there are curves matching the hypothesis of the theorem: two smooth conics with only one intersection point. Using Kashiwara's pencils one can construct many such examples. For instance, one can take two generic elements of a pencil whose generic member is a quartic with an $\mathbb{A}_{6}$ singularity. Other examples are obtained by means of the pencil generated by the square of a quintic curve with an $\mathbb{A}_{12}$ singularity and the fifth power of a conic with highest contact with the quintic at the singular point. All the other curves of the pencil are generic and have only one singular point with local equation $x^{4}-y^{25}=0$. Any curve having at least two members of the pencil as components (at least one generic and at most one special) has a non-zero residue.

\section{Monodromy conjecture for SIS}

From the previous section we learned that, in order to prove the monodromy conjecture for SIS, we have to study the candidate pole $s_{0}=-3 / d$ for those SIS whose tangent cone $C_{d}$ has all its components in a pencil $\Lambda$ of type $(0,1)$ verifying the conditions $(N-1)$ or $(N-2)$ :

$(N-1)$ The divisor $C_{d}$ verifies $\chi\left(\mathbb{P}^{2} \backslash C_{d}\right)=0, s_{0}=-3 / d$ is not a pole for the local DenefLoeser zeta function at any singular point in $C_{d}$ and $\rho\left(C_{d}\right) \neq 0$. In fact, since its components are in a pencil of type $(0,1), C_{d}$ has only one singular point $P$. Furthermore, $s_{0}$ is a pole if and only if $\rho\left(C_{d}\right) \neq 0$. In this case it is a simple pole and the curve $C_{d}$ has only two irreducible components at least one of them being a generic fiber of the pencil. According to the formula for the characteristic polynomial of the complex monodromy of the SIS, we have to prove that $\exp (-2 \mathrm{i} \pi(3 / d))$ is a root of the Alexander polynomial of the germ of $C_{d}$ at the singular point $P$. 
$(N-2) \chi\left(\mathbb{P}^{2} \backslash C_{d}\right)<0$. Let $r$ be the number of irreducible components of $C_{d}(r>2$ and $\left.\chi\left(\mathbb{P}^{2} \backslash C_{d}\right)=2-r\right)$. Since $\rho\left(C_{d}\right)$ is related with the residue of the Denef-Loeser zeta function at $-3 / d$ we distinguish two cases:

- If $s_{0}$ is a simple pole the above discussion also holds, and hence, we must prove that $\exp (-2 \mathrm{i} \pi(3 / d))$ is a root of multiplicity at least $(r-1)$ of the Alexander polynomial of $C_{d}$ at $P$.

- If $s_{0}$ is a multiple pole, then $s_{0}=-3 / d$ is a pole of the local Denef-Loeser zeta function at the singular point $P$. In this case, the irreducible components of $C_{d}$ can also be non-generic fibers of the pencil (since the residue can be equal to or different from zero). We will prove that $\exp (-2 \mathrm{i} \pi(3 / d))$ is a root of multiplicity at least $(r-1)$ of the Alexander polynomial of $C_{d}$ at $P$.

The pencil $\Lambda$ is defined by a rational function $R_{l}$ on $\mathbb{P}^{2}$. Pencils of type $(0,1)$ have at most two special members. We denote them by $\left\{P_{l}=0\right\}$ and $\left\{Q_{l}=0\right\}-$ see Appendix A.

THEOREM 3.1. - The monodromy conjecture is true for SIS singularities.

After all our previous analysis the above theorem is a consequence of the following result.

THEOREM 3.2. - Let $D$ be a divisor of degree $d$ on $\mathbb{P}^{2}$ whose support is the curve $C_{1} \cup C_{2}$, where (1) $C_{1}$ is the union of any number of different generic members $\left\{R_{l}=\mu_{i}\right\}$ of the pencil defined by $R_{l}$ and (2) $C_{2}$ is one of the curves $\left\{P_{l}=0\right\},\left\{Q_{l}=0\right\},\left\{P_{l} Q_{l}=0\right\}$ or $\left\{R_{l}=\mu\right\}$, $\mu \neq \mu_{i}$. Let $r$ be the number of irreducible components of $D$.

(1) If $r \geqslant 3$ then $\exp (-2 \mathrm{i} \pi(3 / d))$ is a root of the Alexander polynomial of the germ of $D$ at its singular point, and its multiplicity is at least $(r-1)$.

(2) Otherwise, if $\rho(D) \neq 0$, then $\exp (-2 \mathrm{i} \pi(3 / d))$ is a root of the Alexander polynomial of the germ of $D$ at its singular point.

In fact, the result will be proved for a (not necessarily reduced) divisor $D$, and used in the following section.

The Alexander polynomial of the complex monodromy of a germ of a plane curve singularity is equal to the Alexander polynomial of its splice diagram $\mathcal{D}$. Let $l_{v}$ be the multiplicity of the vertex $v$ and $\delta_{v}$ its valence. According to [13, p. 96], the Alexander polynomial of a diagram $\mathcal{D}$ is

$$
\Delta_{\mathcal{D}}(t)=(t-1) \prod_{v}\left(t^{l_{v}}-1\right)^{\delta_{v}-2}
$$

the product being taken over all the vertices of the diagram.

Remark 3.3. - Let $v$ be a vertex of valence 1 connected to a vertex $v^{\prime}$ of valence greater than or equal to 3 . Then

$$
\frac{t^{l_{v^{\prime}}}-1}{t^{l_{v}}-1}
$$

is a polynomial, since $l_{v}$ divides $l_{v^{\prime}}$.

Proof of the theorem. - Let $n$ be the number of irreducible components of the curve $C_{1}$. We have four different cases to consider, depending on the irreducible components of the curve $C_{2}$.

- The irreducible component of $C_{2}$ is $\left\{P_{l}=0\right\}$.

- The irreducible component of $C_{2}$ is $\left\{Q_{l}=0\right\}$.

- The irreducible component of $C_{2}$ is $\left\{R_{l}=\mu\right\}$.

- The irreducible components of $C_{2}$ are $\left\{P_{l}=0\right\}$ and $\left\{Q_{l}=0\right\}$.

We use Kashiwara's classification of type $(0,1)$-pencils and divide the proof of the theorem in several steps. 


\subsection{Rational pencils of type $(0,1)$ belonging to $\mathcal{F}_{I I}$}

We begin by studying the set $\mathcal{F}_{I I}$ of pencils of type $(0,1)$. From Appendix A, in the case $\mathcal{F}_{I I}$ one has two splice diagrams. The diagrams only appear with the decorations needed in our computations. The first one $\mathbf{1}$ is shown on the left in Fig. 3 and the second one $\mathbf{2}$ is shown on the right, where $m \in\left\{m_{l+1}, m_{l-1}\right\}$. We need the integer $c$ defined as the product of the numbers which are attached to the non-arrowed vertical edges. On the righthand side of Fig. 3, let $c$ be the product of the numbers which are attached either to the non-arrowed vertical edges or to the last edge on the right.

For each one of the above types of splice diagrams we have four different cases to consider, depending on the irreducible components of the curve $C_{2}$.

CASE 1. - The irreducible component of $C_{2}$ is $\left\{P_{l}=0\right\}$.

The splice diagram 1 is shown in Fig. 4. Because of the preceding remark there exists a polynomial $H(t) \in \mathbb{C}[t]$ such that

$$
\Delta(t)=H(t) \frac{\left(t^{L}-1\right)^{n}}{t^{L^{\prime}}-1}
$$

where $L$ is the multiplicity of the vertex at which the generic fibers $\left\{R_{l}=\mu_{i}\right\}$ separate and $L^{\prime}$ is the multiplicity at the right end of the diagram.

We have the following equalities

$$
d=\left(k_{1}+\cdots+k_{n}\right) \operatorname{deg}\left(R_{l}\right)+k \operatorname{deg}\left(P_{l}\right)=\operatorname{deg}\left(P_{l}\right)\left(\left(k_{1}+\cdots+k_{n}\right) m_{l}+k\right) .
$$

To compute $L$ we use the fact that the intersection multiplicity of two generic curves of the pencil is $m_{l}^{2} \operatorname{deg}\left(P_{l}\right)^{2}$ and the intersection multiplicity of a generic curve of the pencil with $\left\{P_{l}=0\right\}$ is $m_{l} \operatorname{deg}\left(P_{l}\right)^{2}$. Hence,

$$
L=\left(k_{1}+\cdots+k_{n}\right) m_{l}^{2} \operatorname{deg}\left(P_{l}\right)^{2}+k m_{l} \operatorname{deg}\left(P_{l}\right)^{2} .
$$

Therefore $L=d m_{l} \operatorname{deg}\left(P_{l}\right)$. Note that $L^{\prime}=c m_{l}\left(\left(k_{1}+\cdots+k_{n}\right) m_{l}+k\right)$ and $c$ can be computed using $m_{l} \operatorname{deg}\left(P_{l}\right)=m_{l} m c$. Thus,

$$
L^{\prime}=\frac{m_{l}\left(\left(k_{1}+\cdots+k_{n}\right) m_{l}+k\right) \operatorname{deg}\left(P_{l}\right)}{m} .
$$

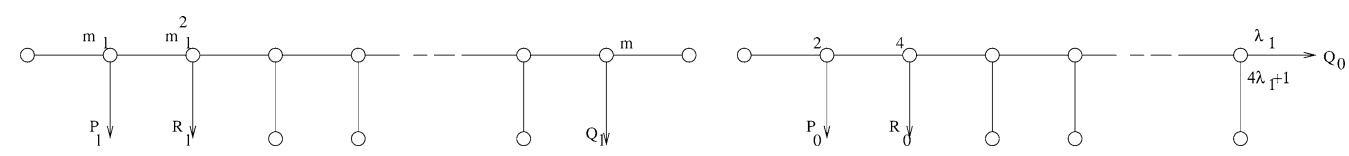

Fig. 3.

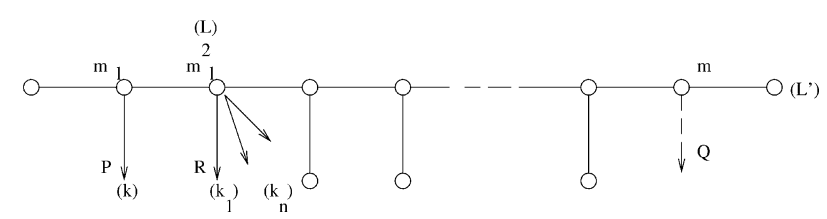

Fig. 4. 
Assume that $(3 / d) L^{\prime} \in \mathbb{N}$, then

$$
\frac{m_{l}\left(\left(k_{1}+\cdots+k_{n}\right) m_{l}+k\right) \operatorname{deg}\left(P_{l}\right)}{m}=\frac{h\left(\left(k_{1}+\cdots+k_{n}\right) m_{l}+k\right) \operatorname{deg}\left(P_{l}\right)}{3},
$$

that is, $3 m_{l}=h m$. Since $\operatorname{gcd}\left(m_{l}, m\right)=1$ and $\operatorname{gcd}(m, 3)=1$, this is not possible, and thus, it proves the theorem in this case.

The splice diagram $\mathbf{2}$ is shown in Fig. 5. The first part of the computation is analogous to the previous case. We still have $L=2 d \operatorname{deg}\left(P_{0}\right)$. One can calculate $L^{\prime}$ as

$$
L^{\prime}=\left(4 \lambda_{1}+1\right) c\left(2 k+4\left(k_{1}+\cdots+k_{n}\right)\right),
$$

and compute the intersection number between $Q_{0}$ and $P_{0}$ as $2\left(4 \lambda_{1}+1\right) c=2 \operatorname{deg}\left(P_{0}\right)$. Thus $L^{\prime}=2 d$ and we cannot conclude the proof. Note that we also have

$$
\Delta(t)=H(t) \frac{\left(t^{L}-1\right)^{n}}{t^{L^{\prime \prime}}-1}
$$

and

$$
L^{\prime \prime}=\lambda_{1} c\left(2 k+4\left(k_{1}+\cdots+k_{n}\right)\right)=\frac{2 \lambda_{1} d}{\left(4 \lambda_{1}+1\right)} .
$$

Finally, if we assume that $(3 / d) L^{\prime \prime} \in \mathbb{N}$ we obtain another contradictory equation $h\left(4 \lambda_{1}+1\right)=$ $6 \lambda_{1}$.

CASE 2. - The irreducible component of $C_{2}$ is $\left\{Q_{l}=0\right\}$.

The splice diagram 1 is shown in Fig. 6. In this case, we have

$$
\Delta(t)=H(t) \frac{\left(t^{L}-1\right)^{n}}{t^{L / m_{l}^{2}}-1}
$$

where, again, $H(t) \in \mathbb{C}[t]$ is a polynomial.

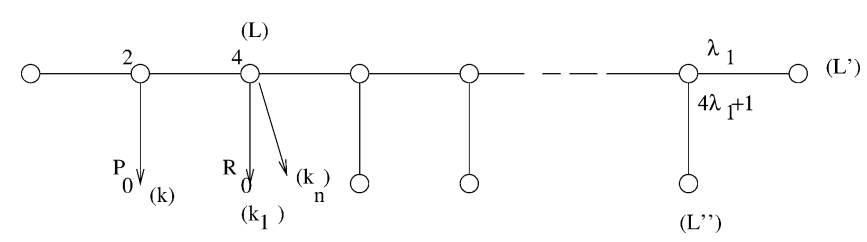

Fig. 5.

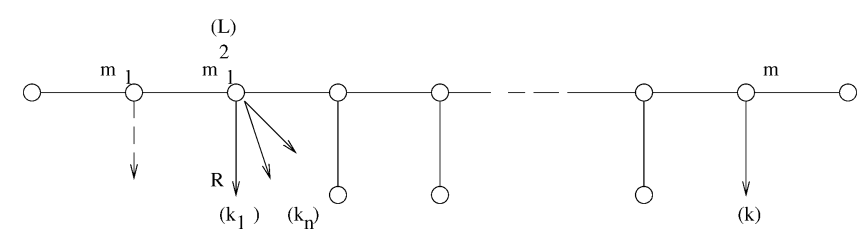

Fig. 6. 


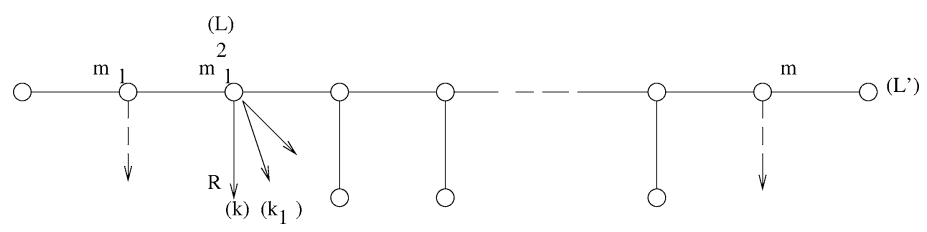

Fig. 7.

We have the following equalities

$$
d=\left(k_{1}+\cdots+k_{n}\right) \operatorname{deg}\left(P_{l}\right) m_{l}+k m_{l}=m_{l}\left(\left(k_{1}+\cdots+k_{n}\right) \operatorname{deg}\left(P_{l}\right)+k\right),
$$

and

$$
L=\left(k_{1}+\cdots+k_{n}\right) \operatorname{deg}\left(P_{l}\right)^{2} m_{l}^{2}+k \operatorname{deg}\left(P_{l}\right) m_{l}^{2} .
$$

If $(3 / d) \cdots \frac{L}{m_{l}^{2}} \in \mathbb{N}$, then

$$
\operatorname{deg}\left(P_{l}\right)\left(\left(k_{1}+\cdots+k_{n}\right) \operatorname{deg}\left(P_{l}\right)+k\right)=h m_{l} \frac{\left(k_{1}+\cdots+k_{n}\right) \operatorname{deg}\left(P_{l}\right)+k}{3},
$$

that is, $3 \operatorname{deg}\left(P_{l}\right)=h m_{l}$. Note again that $\operatorname{gcd}\left(\operatorname{deg}\left(P_{l}\right), m_{l}\right)=1$ and $\operatorname{gcd}\left(m_{l}, 3\right)=1$. The computation is analogous for splice diagram 2.

CASE 3. - The irreducible component of $C_{2}$ is $\left\{R_{l}=\mu\right\}$.

The splice diagram $\mathbf{1}$ is shown in Fig. 7. The Alexander polynomial is

$$
\Delta(t)=H(t) \frac{\left(t^{L}-1\right)^{n+1}}{\left(t^{L / m_{l}^{2}}-1\right)\left(t^{L^{\prime}}-1\right)} .
$$

Analogously, we obtain the following equalities $d=\left(k+k_{1}+\cdots+k_{n}\right) m_{l} \operatorname{deg}\left(P_{l}\right)$, and

$$
\begin{gathered}
L=\left(k+k_{1}+\cdots+k_{n}\right) m_{l}^{2} \operatorname{deg}\left(P_{l}\right)^{2}=\left(k+k_{1}+\cdots+k_{n}\right) c m_{l}^{2}, \\
L^{\prime}=\operatorname{deg}\left(P_{l}\right) m_{l}^{2} \frac{k+k_{1}+\cdots+k_{n}}{m} .
\end{gathered}
$$

Assume that $(3 / d) \cdot \frac{L}{m_{l}^{2}} \in \mathbb{N}$. Thus,

$$
\left(k+k_{1}+\cdots+k_{n}\right) \operatorname{deg}\left(P_{l}\right)^{2}=h \operatorname{deg}\left(P_{l}\right) m_{l} \frac{k+k_{1}+\cdots+k_{n}}{3},
$$

that is, $3 \operatorname{deg}\left(P_{l}\right)=h m_{l}$ which is impossible. If $(3 / d) L^{\prime} \in \mathbb{N}$, then

$$
\operatorname{deg}\left(P_{l}\right) m_{l}^{2} \frac{k+k_{1}+\cdots+k_{n}}{m}=h m_{l} \operatorname{deg}\left(P_{l}\right) \frac{k+k_{1}+\cdots+k_{n}}{3} .
$$

This gives $3 m_{l}=h m$ which is impossible. The theorem is, hence, proved in this case.

The splice diagram $\mathbf{2}$ is shown in Fig. 8. We can write the Alexander polynomial as

$$
\Delta(t)=H(t) \frac{\left(t^{L}-1\right)^{n+1}}{\left(t^{L / 4}-1\right)\left(t^{L^{\prime \prime}}-1\right)} .
$$




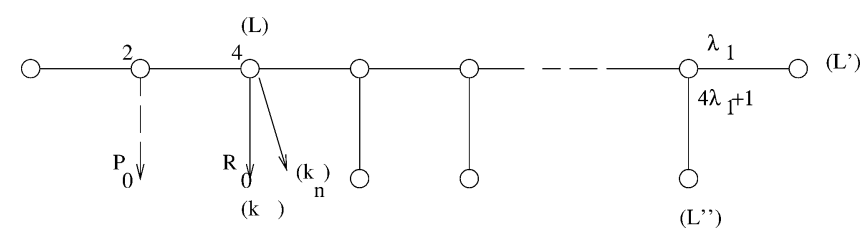

Fig. 8.
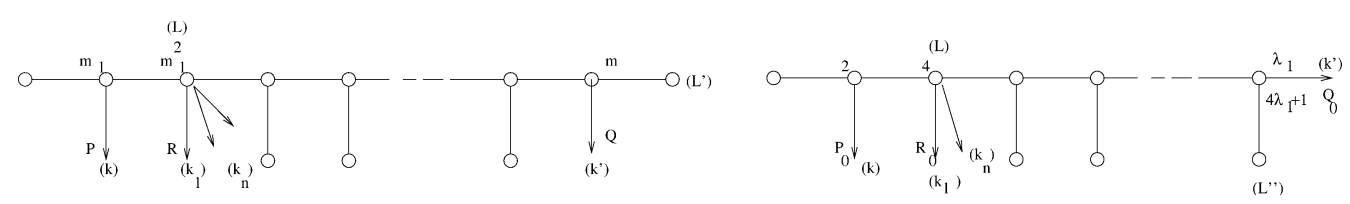

Fig. 9.

The computation of $L$ is the same as above. The computation of $L^{\prime \prime}$ gives

$$
L^{\prime \prime}=4 \lambda_{1} c\left(k+k_{1}+\cdots+k_{n}\right) .
$$

Note that $2\left(4 \lambda_{1}+1\right) c=2 \operatorname{deg}\left(P_{0}\right)$. Thus,

$$
L^{\prime \prime}=4 \lambda_{1} \frac{k+k_{1}+\cdots+k_{n}}{4 \lambda_{1}+1} .
$$

If $(3 / d) L^{\prime \prime} \in \mathbb{N}$, one has $3 \lambda_{1}=h\left(4 \lambda_{1}+1\right)$ with $h \in \mathbb{N}$, which is impossible.

CASE 4. - The irreducible components of $C_{2}$ are $\left\{P_{l}=0\right\}$ and $\left\{Q_{l}=0\right\}$.

Note that in this case the number of irreducible components of $C_{1}$ is $n \geqslant 1$, because we are in the case $(N-2)$ with multiple pole. The splice diagram $\mathbf{1}$ is shown on the lefthand side of Fig. 9. The Alexander polynomial of the local singularity is

$$
\Delta(t)=H(t)\left(t^{L}-1\right)^{n}
$$

where $H \in \mathbb{C}[t]$ is a polynomial. The following equalities hold

$$
\begin{gathered}
L=m_{l}^{2} \operatorname{deg}\left(P_{l}\right)^{2}\left(k_{1}+\cdots+k_{n}\right)+k m_{l} \operatorname{deg}\left(P_{l}\right)^{2}+k^{\prime} m_{l}^{2} \operatorname{deg} P_{l}, \\
d=k \operatorname{deg}\left(P_{l}\right)+\left(k_{1}+\cdots+k_{n}\right) m_{l} \operatorname{deg}\left(P_{l}\right)+k^{\prime} m_{l} .
\end{gathered}
$$

Then $L=m_{l} \operatorname{deg}\left(P_{l}\right) d$.

The splice diagram 2 is shown on the righthand side of Fig. 9. Computations are the same. Then we are done for the case where the corresponding rational function is in $\mathcal{F}_{I I}$.

Example 3.4. - Computing the value for $s_{0}=-3 / d$ in the examples given in Appendix A, one sees that, in fact, $s_{0}=-3 / d$ is a pole of the local Denef-Loeser zeta function of the corresponding SIS singularity.

For example, for

$$
Q_{0}=y-x^{2}, \quad P_{0}=\left(y-x^{2}\right)^{2}-2 x y^{2}\left(y-x^{2}\right)+y^{5} .
$$




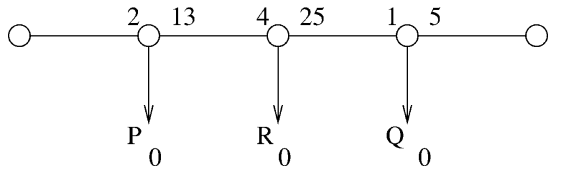

Fig. 10.

Its splice diagram is shown in Fig. 10. The local Denef-Loeser zeta function of the curve $D=\left\{P_{0}=0\right\} \cup\left\{R_{0}=\mu\right\}$ at the origin is

$$
\begin{aligned}
Z_{\mathrm{top}, 0}(D, s)= & \frac{25}{29+150 s}+\frac{2}{(29+150 s)(15+76 s)}+\frac{2}{15+76 s}-\frac{1}{29+150 s} \\
& -\frac{1}{15+76 s}+\frac{1}{(29+150 s)(1+s)}+\frac{1}{(15+76 s)(1+s)}
\end{aligned}
$$

and $Z_{\text {top }, 0}(D,-3 / d)+2 d /(d-3)=-51 / 4$. The Alexander polynomial of the curve $D$ at its only singular point is

$$
\Delta(t)=(t-1) \frac{\left(t^{150}-1\right)\left(t^{76}-1\right)}{\left(t^{6}-1\right)\left(t^{38}-1\right)} .
$$

We will come back to this example in Section 5.

\subsection{Rational pencils of type $(0,1)$ belonging to $\mathcal{F}_{I}$}

We next compute the Alexander polynomial in case $\mathcal{F}_{I},-$ see Appendix A. For this purpose, families $I(0)$ and $I^{+}\left(N ; \lambda_{1}, \ldots, \lambda_{N}\right)$ are considered simultaneously.

- The splice diagram for Case 1 and Case 3 is shown in Fig. 11.

Its Alexander polynomial is

$$
\Delta(t)=H(t) \frac{\left(t^{L}-1\right)^{n}}{t^{L^{\prime}}-1}
$$

where $H(t)$ is a polynomial. One has $L=m d$ and $L^{\prime}=d / 2$. Thus $\exp (-6 \mathbf{i} \pi / d)$ is a root of multiplicity $n$ of the Alexander polynomial.

- The splice diagram for Case 2 and Case 4 is shown in Fig. 12.

One has $\Delta(t)=H(t)\left(t^{L}-1\right)^{n-1}$. Thus $L=m d$. This implies that the conjecture is verified in this case if $n \neq 1$.

The remaining family in $\mathcal{F}_{I}$ is $I^{-}\left(N ; \lambda_{1}, \ldots, \lambda_{N}\right)$.

- The splice diagram for Case 1 and Case 3 is shown in Fig. 13.

One has

$$
\Delta(t)=H(t) \frac{\left(t^{L}-1\right)^{n}}{t^{L^{\prime}}-1}, \quad \text { or } \quad \Delta(t)=H_{1}(t) \frac{\left(t^{L}-1\right)^{n}}{t^{L^{\prime \prime}}-1} .
$$

We still have $L=m d$, but we now have $L^{\prime}=d$ and $L^{\prime \prime}=d \frac{\lambda_{1}}{\lambda_{1}+1}$. If $\lambda_{1}=2$ and there exists only one component, i.e. $n=1$, then $\exp (2 \mathrm{i} \pi(-3 / d))$ is not a root of the Alexander polynomial.

In order to prove the monodromy conjecture in the two remaining cases we have to prove that the bad divisor $D$ verifies $\rho(D)=0$. The curve $D$ consists of (at least) two generic members of a pencil of type $I^{-}\left(N ; 2, \ldots, \lambda_{N}\right)$.

From its resolution graph - see Appendix A - one can check that the only possible contraction to the ruled surface $\Sigma$ in these cases is the blowing-down of the line $\{Q=0\}$. The curve $\{Q=0\}$ is the only special fiber of these pencils, it is also a multiple fiber. In any case, it is readily checked 

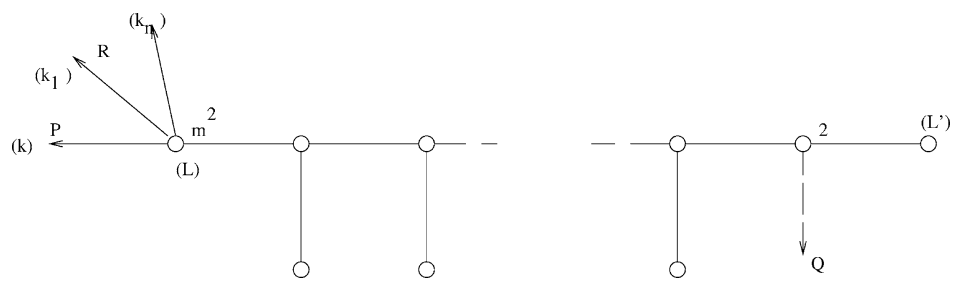

Fig. 11 .
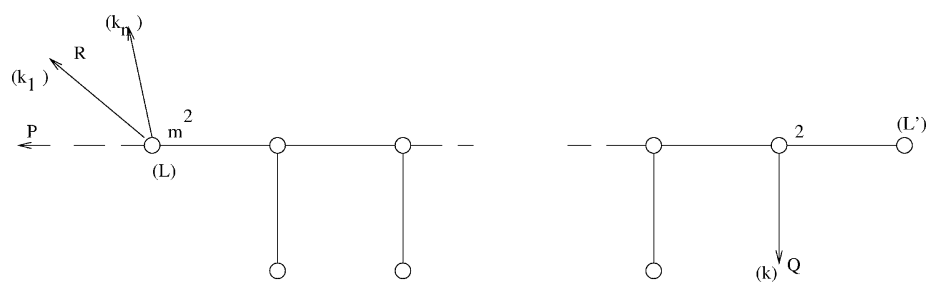

Fig. 12.
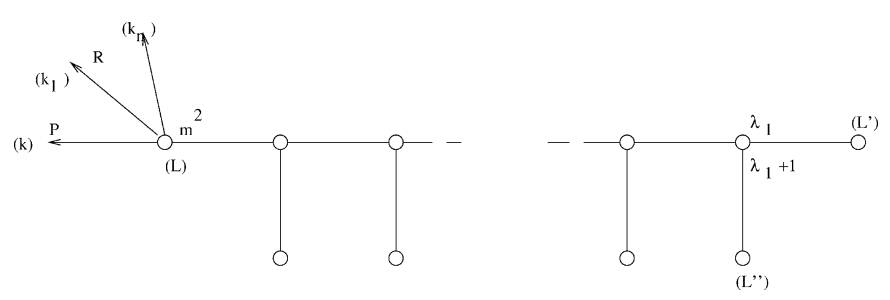

Fig. 13.

that the map $\pi_{2}$ is the identity (one resolves the pencil when resolving the curve, since there are at least two generic fibers). The blowing-ups of type (ii) in 2.10 produce special fibers of the pencil. Thus, only one such blowing-up can take place. It is easily seen that the blowing-up producing $\{Q=0\}$ occurs in a component of valency one, and in this case the $\zeta$-invariant does not change and the residue $\rho(D)$ is zero.

- For Case 2 and Case 4 the arguments of the proof are analogous and we leave the proof to the reader.

The theorem is proved.

\section{Monodromy conjecture for homogeneous polynomials}

B. Rodrigues and W. Veys have proved the monodromy conjecture for any homogeneous polynomial $f_{d} \in \mathbb{C}\left[x_{1}, x_{2}, x_{3}\right]$ with $\chi\left(\mathbb{P}^{2} \backslash\left\{f_{d}=0\right\}\right) \neq 0$, see [26].

In fact, in the proof of their Theorem 4.2 they showed that for any homogeneous polynomial $f_{d} \in \mathbb{C}\left[x_{1}, x_{2}, x_{3}\right]$ of degree $d$ and for any pole $s_{0} \neq-3 / d$ of $Z_{\text {top }, 0}(f, s), \exp \left(2 \mathrm{i} \pi s_{0}\right)$ is an eigenvalue of the local monodromy of $f_{d}$ at some complex point of the effective divisor $D=f_{d}^{-1}(0)$.

One of the key points in their proof of the homogeneous case, is the following equality - see $[26,(3.6)]$ 


$$
\begin{aligned}
Z_{\mathrm{top}, 0}\left(f_{d}, s\right) & =\frac{1}{3+d s} z(D, s) \\
& =\frac{1}{3+d s}\left(\chi\left(\mathbb{P}^{2} \backslash D\right)+\sum_{i=1}^{r} \frac{\chi\left(\check{D}_{i}\right)}{1+a_{i} s}+\sum_{P \in \operatorname{Sing}\left(D_{\mathrm{red}}\right)} Z_{\mathrm{top}, P}(D, s)\right),
\end{aligned}
$$

where $D=a_{1} D_{1}+\cdots+a_{r} D_{r}$ and $\check{D}_{i}:=D_{i} \backslash \operatorname{Sing}\left(D_{\text {red }}\right)$.

We are interested in the remaining case $\chi\left(\mathbb{P}^{2} \backslash\left\{f_{d}=0\right\}\right)=0$ and the candidate pole $s_{0}=-3 / d$. If $s_{0}$ is a pole of order greater than 1 , then either $s_{0}=-3 / d$ is a pole of $Z_{\text {top, } P}(D, s)$ (and thus the monodromy conjecture for curves implies that $\exp \left(2 \mathrm{i} \pi s_{0}\right)$ is a root of the local monodromy of $f_{d}$ at some complex point of $D$ ) or $s_{0}=-3 / d$ is the pole $-1 / a_{i}$, for some $a_{i}$. In such a case, if $P \in D_{i} \cap \operatorname{Sing}(D) \neq \emptyset$ then the branches of $D$ at $P$ have multiplicity $a_{i}$ and $\mathrm{W}$. Veys showed that $Z_{\mathrm{top}, P}(D, s)$ has $-1 / a_{i}$ as a pole. Again, the monodromy conjecture for curves implies that $\exp \left(2 \mathrm{i} \pi s_{0}\right)$ is a root of the local monodromy of $f_{d}$ at some complex point of $D$.

The discussion above translates into the following: $s_{0}=-3 / d$ is a simple pole of $Z_{\text {top }, 0}\left(f_{d}, s\right)$ if and only if $D$ is a bad divisor on $\mathbb{P}^{2}$ and $z(D,-3 / d)=\rho(D) \neq 0, \infty$. However, according to Theorem $3.2 \exp (2 \mathrm{i} \pi(-3 / d))$ is an eigenvalue of the monodromy of its only singular point. Thus, the monodromy conjecture is also proved in this remaining case. The results by B. Rodrigues and W. Veys and the above discussion show the following theorem.

THEOREM 4.1.-For any homogeneous polynomial $f_{d} \in \mathbb{C}\left[x_{1}, x_{2}, x_{3}\right]$ the monodromy conjecture holds.

\section{Rational arrangements of plane curves}

The results of this paper can be applied to prove the non-existence of some arrangements of rational curves in $\mathbb{P}^{2}$. For such a reason we restrict ourselves to arrangements whose complement in the plane has Euler-Poincare characteristic 0 .

Let $D=\bigcup C_{i}$ be an arrangement of reduced rational curves. The dual graph of the minimal embedded resolution of $D$ is determined by the following data:

(1) The degrees $d_{i}$ of the irreducible components of $D$.

(2) The list of the Denef-Loeser types of the local singularities of $D$.

(3) The irreducible component of $D$ which contains each branch $\Gamma$ of $D$ at a singular point.

We call these data the combinatorial type of the curve $D$ in $\mathbb{P}^{2}$. We also call the data in (2) together with the total degree $d$ of $D$ the local combinatorial data of $D$ in $\mathbb{P}^{2}$.

As a corollary of Theorem 2.15 and Theorem 3.1 one obtains the following result, which is equivalent to the monodromy conjecture for SIS.

Corollary 5.1. - Let $D$ be a bad divisor of degree d on $\mathbb{P}^{2}$. If $\rho(D) \neq 0$ then $D$ has only one singular point and $\exp (2 \mathrm{i} \pi(-3 / d))$ is an eigenvalue of the complex monodromy at that singular point.

Given a divisor $D$ on $\mathbb{P}^{2}$ and a point $P \in D$, the local Denef-Loeser zeta function $Z_{\text {top }, P}(D, s)$, the residue $\rho(D)$ and the eigenvalues of the complex algebraic monodromy of $(D, P)$ are determined by the local combinatorial data of $D$. Hence the monodromy conjecture gives necessary conditions on the local combinatorial data of $D$ for $D$ to exist.

We have developed a program with MapleV (available upon request) which calculates the local embedded resolution of the singularities of a curve $D$, the local Denef-Loeser zeta function $Z_{\text {top }, P}(D, s)$, the residue $\rho(D)$ and the eigenvalues of the complex algebraic monodromy of 
$(D, P)$ from the local combinatorial data of $D$. Thus, given local combinatorial data of $D$ the above necessary conditions can be easily verified. Let us present some few examples.

Example 5.2. - Let $D$ consist of two conics which only meet at one point and a line which is tangent to each conic in different points. Using elementary properties of pencils of conics it is easy to see that $D$ does not exist. In this case, the residue $\rho(D)$ would be $-3 / 5$ (different from 0 ) but there would be three singular points. Thus it would give a counterexample to the above corollary.

Example 5.3. - Consider a rational curve $C$ of degree six with only one singular point $P$ which is a simple singularity. Then $P$ can be either an $\mathbb{A}_{19}$ or $\mathbb{A}_{20}$ singularity. It is known that the $\mathbb{A}_{19}$ case exists, e.g. see [34]. The double covering of $\mathbb{P}^{2}$ ramified along $C$ is a $K 3$-surface. Using $K 3$-surface theory one shows that the $\mathbb{A}_{20}$ case is not possible.

Let $D=C \cup C_{2}$ be the curve whose components are the sextic $C$ with the $\mathbb{A}_{20}$ singularity at $P$ and $C_{2}$, where the latter is the unique conic passing through the first five infinitely near points of $C$ at $P$. We suppose that this conic in fact passes through the sixth infinitely near point of $C$ at $P$. Hence the conic only meets $C$ at its singular point. The residue $\rho(D)$ would be different from 0 and the characteristic polynomial of the monodromy of $(D, P)$ would turn out to be

$$
\Delta_{D, P}(t)=\frac{(t-1)\left(t^{17}-1\right)\left(t^{54}-1\right)}{\left(t^{27}-1\right)\left(t^{3}-1\right)} .
$$

Hence $D$ does not exist because $\exp (2 \mathrm{i} \pi(-3 / 8))$ is not an eigenvalue of the complex monodromy of $D$ at $P$.

Example 5.4. - Consider $C$ a rational curve of degree 10 with only one singular point $P$ whose multiplicity sequence is $[4,4,4,4,4,4,1,1,1,1]=\left[4_{6}\right]$ (this curve exists and it appears in the classification of $\mathrm{H}$. Kashiwara, see Appendix A).

Let $D=C \cup C_{2}$ be the curve whose components are $C$ and $C_{2}$, where the latter is the unique conic passing through the first five infinitely near point of $C$ at $P$; by Bezout's theorem there is no other intersection point. In this case the residue $\rho(D)=-3$ and $\exp (2 \mathrm{i} \pi(-3 / 12))$ is a root of the characteristic polynomial of the monodromy of $D$ at $P$. Its Alexander polynomial is the following:

$$
\Delta_{D, P}(t)=\frac{(t-1)\left(t^{25}-1\right)\left(t^{120}-1\right)}{\left(t^{5}-1\right)\left(t^{30}-1\right)} .
$$

The following is a list of several possible cuspidal rational curves of degree 10 which might exist. We give each singularity as a sequence of multiplicities.

$$
\begin{array}{cccc}
{\left[4_{5}, 2_{6}\right],} & {\left[4_{5}, 2_{5}\right]+1 \mathbb{A}_{2},} & {\left[4_{5}, 2_{4}\right]+2 \mathbb{A}_{2},} & {\left[4_{5}, 2_{4}\right]+1 \mathbb{A}_{4},} \\
{\left[4_{5}, 2_{3}\right]+3 \mathbb{A}_{2},} & {\left[4_{5}, 2_{3}\right]+3 \mathbb{A}_{2},} & {\left[4_{5}, 2_{3}\right]+1 \mathbb{A}_{2}+1 \mathbb{A}_{4},} & {\left[4_{5}, 2_{3}\right]+1 \mathbb{A}_{6},} \\
{\left[4_{5}, 2_{3}\right]+1 \mathbb{E}_{6},} & {\left[4_{5}, 2_{2}\right]+4 \mathbb{A}_{2},} & {\left[4_{5}, 2_{2}\right]+2 \mathbb{A}_{2}+1 \mathbb{A}_{4},} & {\left[4_{5}, 2_{2}\right]+2 \mathbb{A}_{4},} \\
{\left[4_{5}, 2_{2}\right]+1 \mathbb{A}_{2}+1 \mathbb{A}_{6},} & {\left[4_{5}, 2_{2}\right]+1 \mathbb{A}_{2}+1 \mathbb{E}_{6},} & {\left[4_{5}, 3\right]+1 \mathbb{A}_{2}+1 \mathbb{A}_{4},} & {\left[4_{5}, 3\right]+3 \mathbb{A}_{2},} \\
{\left[4_{5}, 3\right]+1 \mathbb{A}_{6},} & {\left[4_{5}, 3\right]+1 \mathbb{E}_{6},} & {\left[4_{5}, 2_{2}\right]+1 \mathbb{A}_{8} .} &
\end{array}
$$

If one considers the corresponding curve $D$ as the union of the curve of degree 10 with these singularities and the conic as before, then all of them define bad divisors with residue $\rho(D) \neq 0$. Thus all of them but the first one do not exist because they have more than one singular point. In 
fact, the first one would also give a counter-example to the above corollary. The invariants for a SIS $(V, 0)$ whose tangent cone is a curve with such properties are

$$
\begin{gathered}
Z_{\mathrm{top}, 0}(V, s)=\frac{93547584 s^{4}+436242144 s^{3}+294239146 s^{2}+71173441 s+5854275}{7(1+s)(1+4 s)(59+234 s)(81+325 s)(175+702 s)}, \\
\Delta_{V}(t)=\frac{\left(t^{819}+1\right)\left(t^{364}-t^{351}+t^{13}-1\right)\left(t^{260}+t^{195}+t^{130}+t^{65}+1\right)}{(t-1)} .
\end{gathered}
$$

Then $s_{0}=-1 / 4$ is a pole of the Denef-Loeser zeta function but $-\mathrm{i}=\exp \left(2 \mathrm{i} \pi \frac{-1}{4}\right)$ is not an eigenvalue of the complex monodromy. Hence such a curve does not exist.

Question. - Suppose one gives a set of local data, the question arises whether there exists a curve satisfying such combinatorics. In this situation we will say we have a potential curve. Note that concepts such as rationallity, bad divisor or residue at a singular point only depend on the combinatorics, so we can talk about these concepts for a potential curve. Hence, Corollary 5.1 gives a necessary condition for a potential rational bad divisor $D$ with $\rho(D) \neq 0$ to exist, namely $\exp \left(2 \mathrm{i} \pi \frac{-3}{d}\right)$ is an eigenvalue of the complex monodromy at its singular point.

An interesting question is whether such condition is also sufficient or not, that is, for any combinatorial data for a potential bad divisor $D$ with $\rho(D) \neq 0$ if $\exp \left(2 \mathrm{i} \pi \frac{3}{d}\right)$ is an eigenvalue of the complex monodromy at its singular point then a curve with these data always exists.

\section{On a question by $D$. Siersma}

Throughout this section let $f_{d} \in \mathbb{C}\left[x_{1}, x_{2}, x_{3}\right]$ be a degree $d$ homogeneous polynomial such that the projective curve $D=f_{d}^{-1}(0) \subset \mathbb{P}^{2}$ is reduced. Because of the homogeneity condition, $f_{d}$ defines a local and a global object in the following sense.

D. Siersma in [27] studied singularities with a 1-dimensional critical locus. In particular his results can be applied to the Milnor fibration of the germ $f_{d}:\left(\mathbb{C}^{3}, 0\right) \rightarrow(\mathbb{C}, 0)$. Its Milnor fiber $F$ has non-vanishing homology groups concentrated in dimensions 0,1 and 2 . In fact, $H_{2}(F, \mathbb{Z})$ is a free group and $H_{1}(F, \mathbb{Z})$ can have torsion. One has the algebraic monodromy acting on $F$ :

$$
T_{i}: H_{i}(F, \mathbb{Z}) \rightarrow H_{i}(F, \mathbb{Z})
$$

Since $f_{d}$ is homogeneous, the Milnor fibration can be extended to $\mathbb{C}^{n} \backslash f_{d}^{-1}(0)$. This has been studied by A. Dimca in [11]. From [27] or [11] it is known that a necessary condition for $T_{2}$ to be equal to the identity, $T_{2}=1$, is $\chi\left(\mathbb{P}^{2} \backslash D\right)=0$. It was an open question if this condition is also a sufficient condition.

A characterization of such a condition would be of interest after the following theorem of A. Dimca and A. Némethi, [12].

THEOREM A. - For $i=0,1,2$ the following statements are equivalent.

(a) $T_{i}=\mathbf{1}$,

(b) the (reduced) homology monodromy representation

$$
\rho\left(f_{d}\right)_{i}: \pi_{1}\left(\mathbb{C}^{*}, p t\right) \rightarrow \operatorname{Aut}\left(\tilde{H}_{i}(F, \mathbb{Z})\right)
$$

is trivial. 
D. Siersma listed several examples where the condition $\chi\left(\mathbb{P}^{2} \backslash D\right)=0$ was also sufficient. In all of these examples the residue $\rho(D)=0$. Consider Example 2.14, namely $D$ consisting of two conics meeting at only one point $P$ which is an $\mathbb{A}_{7}$ singularity, that is $\operatorname{Sing}(D)=\{P\}$. The curve $D$ has degree 4 and $\chi\left(\mathbb{P}^{2} \backslash D\right)=0$. This example appeared on D. Siersma's list, but his equation is not an $\mathbb{A}_{7}$ singularity.

We are going to prove that $T_{1} \neq \mathbf{1}$ for $f_{d}$ defining $D$, for instance

$$
f_{d}=\left(x_{1} x_{3}-x_{2}^{2}\right)\left(x_{1} x_{3}-x_{2}^{2}+x_{1}^{2}\right) .
$$

This fact and the following well-known formula for the zeta-function of the monodromy of homogeneous polynomials, [1], prove that the condition $\chi\left(\mathbb{P}^{2} \backslash D\right)=0$ does not imply $T_{2}=\mathbf{1}$ :

$$
\frac{\operatorname{det}\left(\mathbf{1}-t T_{0}\right) \operatorname{det}\left(\mathbf{1}-t T_{2}\right)}{\operatorname{det}\left(\mathbf{1}-t T_{1}\right)}=\left(1-t^{4}\right)^{\chi\left(\mathbb{P}^{2} \backslash D\right)}=1 \text {. }
$$

To compute $T_{1}$ we use the method described in [3] - see also [11,14,20,23] for other interesting methods. Let $\pi: X \rightarrow \mathbb{P}^{2}$ be the minimal embedded resolution of the singularity of $D$ at the point $P$. Hence $\pi^{*}(D)=C_{1}+C_{2}+2 E_{1}+4 E_{2}+6 E_{3}+8 E_{4}$ is a normal crossing divisor. Consider for $k=1,2,3$ the ideal sheaf $\mathcal{I}^{k}$ on $\mathbb{P}^{2}$ defined as follows:

- If $Q \in \mathbb{P}^{2} \backslash \operatorname{Sing}(D)$, then $\mathcal{I}_{Q}^{k}=\mathcal{O}_{\mathbb{P}^{2}, Q}$.

- If $P \in \operatorname{Sing}(D)$, then $\mathcal{I}_{P}^{k}$ is the following ideal of $\mathcal{O}_{\mathbb{P}^{2}, P}$ : if $h \in \mathcal{O}_{\mathbb{P}^{2}, P}$, then $h \in \mathcal{I}_{P}^{k}$ if and only if the vanishing order of $\pi^{*}(h)$ along each $E_{i}$ is, at least, $-\left(\nu_{i}-1\right)+\left[\frac{k N_{i}}{4}\right]$ (where [.] stands for the integer part of a real number).

For $l \geqslant 0$, the following map

$$
\sigma_{l, k}: H^{0}\left(\mathbb{P}^{2}, \mathcal{O}(l)\right) \rightarrow \mathcal{O}_{\mathbb{P}^{2}, P} / \mathcal{I}_{P}^{k}: h \mapsto h_{P}+\mathcal{I}_{P}^{k}
$$

is well defined (up to scalars). The following result was proved in [3]:

STATEMENT. - The dimension of the eigen-subspace corresponding to the eigenvalue $\exp (2 \mathrm{i} \pi(k / 4))$ of the algebraic monodromy $T_{1}$ of $D$ is equal to $\operatorname{dim}$ coker $\sigma_{k-3, k}$.

In particular, for $k=3$ one has $H^{0}\left(\mathbb{P}^{2}, \mathcal{O}(0)\right) \cong \mathbb{C}$, and

$$
\left\{-\left(\nu_{i}-1\right)+\left[\frac{3 N_{i}}{4}\right]\right\}_{i=1}^{4}=\{0,1,1,2\} .
$$

Let $x, y$ be local coordinates at $P$ such that $x=0$ is the tangent line of $D$ at $P$. It is easy to see that $\mathcal{I}_{P}^{3}=\left(x, y^{2}\right)$. It turns out that dim coker $\sigma_{0,3}$ is greater than 0 . In particular $T_{1}$ is not the identity.

Question. - For any curve $\left\{f_{d}=0\right\} \subset \mathbb{P}^{2}$ such that $\chi\left(\mathbb{P}^{2} \backslash\left\{f_{d}=0\right\}\right)=0$, it would be interesting to study if there is any relationship between the monodromy conjecture and the fact that the monodromy representation $\rho\left(f_{d}\right)$ is the identity.

\section{Acknowledgements}

During the elaboration of this article, the second author was the host of the Dept. of Algebra at the University Complutense of Madrid supported by a Sabbatical Grant from the MEC. She wishes to thank the MEC for its support and the members of the Dept. of Algebra for their warm 
hospitality. The last three authors thank the Isaac Newton Institute for Mathematical Sciences at Cambridge for its support while finishing this paper at the Institute.

The authors want to thank W. Veys for his useful remarks (see Remark 2.16).

The authors are very grateful to the referee who helped them to improve their work and correct many inaccuracies.

First author is partially supported by DGES PB97-0284-C02-02; third and fourth authors are partially supported by DGES PB97-0284-C02-01.

\section{Appendix A}

Since we are concerned with rational functions on $\mathbb{P}^{2}$ with rational fibers, we review some facts related to this theory. All assertions in this appendix are explained in much greater detail in $[17,18]$.

Let $\Lambda$ be a pencil on the projective plane $\mathbb{P}^{2}$ defined by a non-constant rational function $R$. Let $\left\{p_{1}, \ldots, p_{s}\right\}$ be the set of base points of the pencil. The function $R$ defines a well-defined map $R: \mathbb{P}^{2} \backslash\left\{p_{1}, \ldots, p_{s}\right\} \rightarrow \mathbb{P}^{1}$.

We say that $\Lambda$ (or $R$ ) is of type $(g, n)$ if the irreducible components of a generic fiber of the map $R$ are open Riemann surfaces of genus $g$ with $n$ points on the boundary. A pencil (or a rational function) of type $(0, n)$ is called rational. In addition, if it is of type $(0,1)$ or $(0,2)$ then we say that $\Lambda$ (or $R$ ) is of special type.

H. Kashiwara in [17] (resp. T. Kizuka in [18]) classified the pencils of type $(0,1)$ (resp. pencils of type $(0,2)$ ).

The pencils of type $(0,1)$ on $\mathbb{P}^{2}$ have only one base point $P$, all the members of the pencil are irreducible curves of type $(0,1)$ whose only singular point is $P$, and all the members of the pencil, but at most two, are reduced.

The pencils of type $(0,2)$ on $\mathbb{P}^{2}$ have at most two base points (this will give two sections in the resolution process), one member of the pencil has two irreducible components and the other members are irreducible.

The pencils of special type are classified in two classes: (1) class $\mathcal{F}_{I}$ : all pencils of special type for which there exists a member of the pencil which is a projective line and (2) class $\mathcal{F}_{I I}$ : the pencils not having a line as a member.

In this appendix we collect the graphs for the pencils of type $(0,1)$ obtained by H. Kashiwara. We translate her graphs into Eisenbud-Neumann splice diagrams [13].

Next we recall the required results of H. Kashiwara. They are expressed in terms of the resolution graphs. We will also give them in terms of splice diagrams, which are more convenient for our purposes.

Let $\vec{G}_{l}$ denote the uppermost graph in Fig. 14 if $l=2 j-1, j \geqslant 1$, the one at the bottom lefthand side if $l=2 j, j \geqslant 1$, and the one at the bottom righthand side if $l=0$.

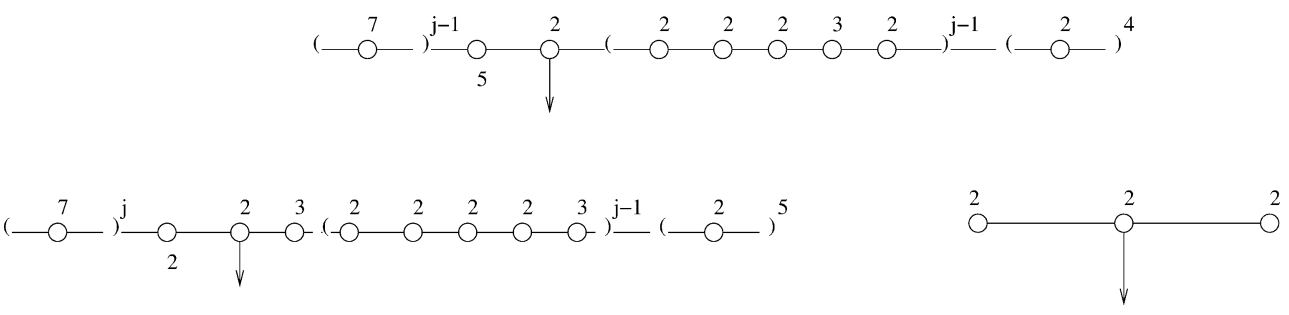

Fig. 14. 
Lemma A.1. - Define $m_{l}, l \in \mathbb{N}$, by

$$
m_{0}=2, \quad m_{1}=5, \quad m_{l}=3 m_{l-1}-m_{l-2} .
$$

If $l=2 j-1, j \geqslant 1$, then $m_{l}$ is the determinant of the graph on the lefthand side of Fig. 15 . If $l=2 j, j \geqslant 1$, then $m_{l}$ is the determinant of the graph on the righthand side of Fig. 15.

Proof. - Denote by $n_{l}$ the determinant of the above graphs. The computation of these determinants is due to N. Duchon and is explained in [13, p. 153]. For $j=1$, it is easy to compute that $n_{1}=5$ and $n_{2}=13$. One easily has the recurrence formula $n_{l}=7 n_{l-2}-n_{l-4}$. The lemma is proved.

H. Kashiwara decomposes $\mathcal{F}_{I I}$ in different sets that will be studied independently. For $l \in \mathbb{N}$, let $R_{l} \in \mathcal{F}_{I I}$ be a rational function given by

$$
R_{l}=\frac{P_{l}^{m_{l}}}{Q_{l}^{\operatorname{deg} P_{l}}} .
$$

Let $\Sigma$ be the resolution graph of the pencil $R_{l}$. Let also $\hat{S}_{0}$ and $\hat{S}_{\infty}$ be the strict transforms of $\left\{P_{l}=0\right\}$ and $\left\{Q_{l}=0\right\}$ respectively. The graph $\Sigma \cup \hat{S}_{0} \cup \hat{S}_{\infty}$ is given in [17, Theorem 6.1, p. 536].

CASE 1. $-I I(l), l \geqslant 0$.

For $l \geqslant 0$, the graph $\Sigma \cup \hat{S}_{0} \cup \hat{S}_{\infty}$ is shown in Fig. 16 .

LEMmA A.2. - The splice diagram of the germ $\left\{P_{l}=0\right\} \cup\left\{R_{l}=\mu\right\} \cup\left\{Q_{l}=0\right\}$ at its singular point is shown in Fig. 17.

Proof. - The strict transform of $\left\{R_{l}=\mu\right\}$ is transversal to the unique component, in the resolution graph, with self-intersection -1 . Using the relation between resolution graphs and splice diagrams as explained in [13], one can check that the corresponding splice diagram is that in Fig. 18.
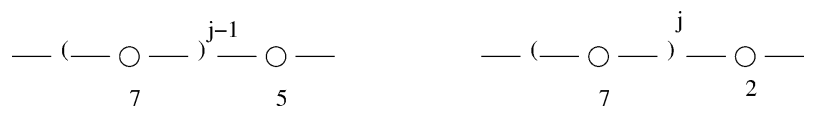

Fig. 15.
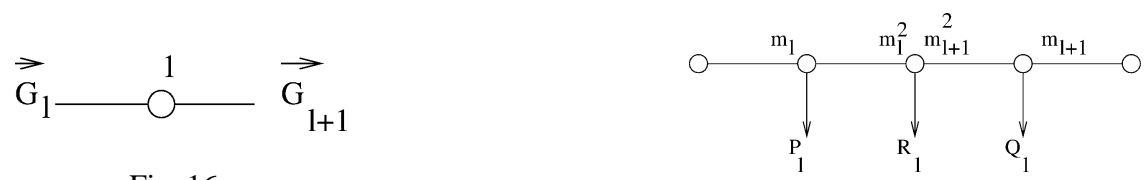

Fig. 16.

Fig. 17.

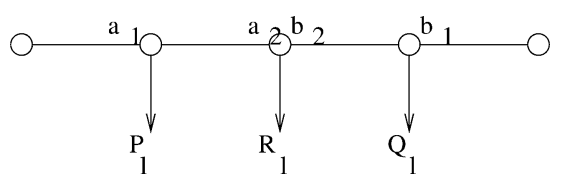

Fig. 18. 
By Lemma A.1, curves $\left\{P_{l}=0\right\}$ and $\left\{Q_{l}=0\right\}$ only meet at $(0,0)$. The computation of intersection multiplicity, which is also explained in [13], yields $a_{1} b_{1}=m_{l} m_{l+1}$ and $b_{1}=m_{l+1}$. Moreover, since $\left\{R_{l}=0\right\}$ is a generic member of the pencil, $a_{2} b_{1} m_{l+1}=a_{1} b_{2} m_{l}$. Since $\operatorname{gcd}\left(m_{l}, m_{l+1}\right)=1$, one has $a_{2}=m_{l}^{2}$ and $b_{2}=m_{l+1}^{2}$. Thus the lemma is proved.

Note that, along the lines, we have also proved the following

LEMMA A.3. - If $l=2 j-1, j \geqslant 1$, then the integer $m_{l}$ is the determinant of the graph on the left-hand side of Fig. 19. If $l=2 j, j \geqslant 1$, then $m_{l}$ is the determinant of the graph on the right-hand side.

The simplest example of rational functions in $I I(l)$ is described in Example 3.4. Its splice diagram can be seen in Fig. 10.

CASE 2. $-I I^{+}\left(l, N ; \lambda_{1}, \ldots, \lambda_{N}\right), l \geqslant 0$.

We denote by $\overleftarrow{G_{l}}$ the graph $\overrightarrow{G_{l}}$ when read from right to left and by $+\overrightarrow{G_{l}}$ the graph $\overrightarrow{G_{l}}$ whose weight at the left end is increased by one. The graph is shown in Fig. 20, where $\lambda_{1}, \ldots, \lambda_{N}$ belong to $\mathbb{Z}_{\geqslant 0}$ if $l \geqslant 1$ and to $\mathbb{Z}_{>0}$ if $l=0$. The $N$-tuple $\left(\lambda_{1}, \ldots, \lambda_{N}\right)$ determines (and it is determined by) the resolution graphs of the vertical sides. We do not use them so we do not need to be more explicit.

LEMmA A.4. - The splice diagram of the germ $\left\{P_{l}=0\right\} \cup\left\{R_{l}=\mu\right\} \cup\left\{Q_{l}=0\right\}$ is shown in Fig. 21.

Proof. - From Lemmas A.1 and A.3, we know that the splice diagram is the one shown in Fig. 22.

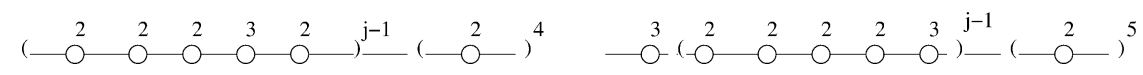

Fig. 19.

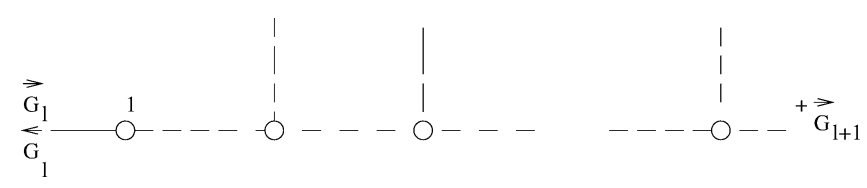

Fig. 20.

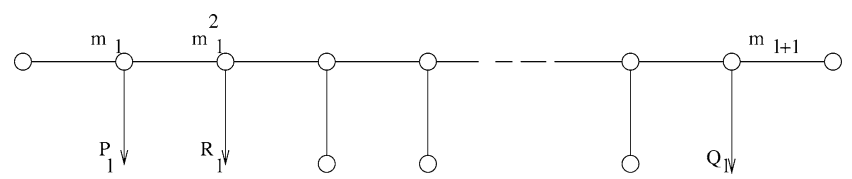

Fig. 21.

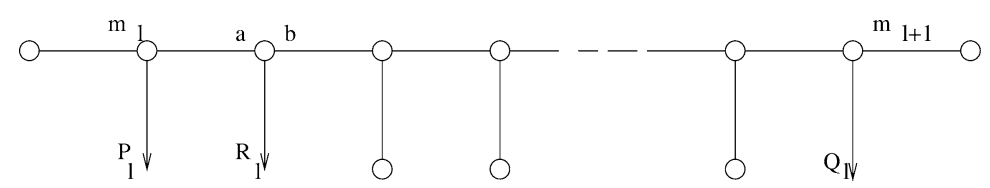

Fig. 22. 
Since $\left\{R_{l}=0\right\}$ is a generic fiber of the pencil, we have $b m_{l}^{2}=a m_{l+1} \operatorname{deg}\left(P_{l}\right)$. Since $\operatorname{gcd}\left(m_{l}, m_{l+1} \operatorname{deg}\left(P_{l}\right)\right)=1$ and $\operatorname{gcd}(a, b)=1$, we have $a=m_{l}^{2}$.

An example of such a rational function is given by the following formulæ. Let

$$
\begin{gathered}
\phi=x y-x^{3}-y^{3}, \quad P_{-1}=y-x^{2}, \quad P_{1}=\left(\phi^{5}+P_{0}^{3}\right) / P_{-1}, \\
F=\phi P_{0}^{2}+a P_{1}, \quad P=\left(F^{5}+P_{0}^{13}\right) / P_{1} .
\end{gathered}
$$

The polynomial $P$ has degree 52 , and is in a $(0,1)$-pencil with $P_{0}$. Its splice diagram is shown in Fig. 23.

CASE 3. $-I I^{-}\left(0, N ; \lambda_{1}, \ldots, \lambda_{N}\right)$.

The resolution graph is shown on the lefthand side of Fig. 24.

LEMmA A.5. - The splice diagram of the germ $\left\{P_{l}=0\right\} \cup\left\{R_{l}=\mu\right\} \cup\left\{Q_{l}=0\right\}$ is shown on the righthand side of Fig. 3.

The proof uses the same argument as above.

One example of such a rational function is

$$
F_{1}=\phi P_{-1}^{2}+a_{3} y P_{-1}^{3}+a_{2} y^{3} P_{-1}^{2}+a_{1} y^{5} P_{-1}+a_{0} y^{7}, \quad P=\left(P_{-1}^{7}+F_{1}^{2}\right) / y .
$$

The polynomial $P$ has degree 13 and is in a $(0,1)$-pencil with $P_{-1}$. Its splice diagram is shown on the righthand side of Fig. 24.

CASE 4. $-I I^{-}\left(l, N ; \lambda_{1}, \ldots, \lambda_{N}\right), l \geqslant 1$.

Its resolution graph is given in Fig. 25.

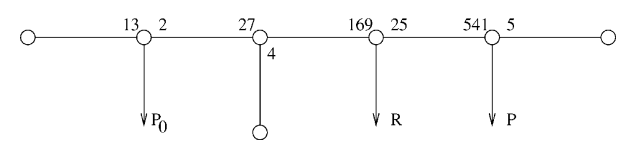

Fig. 23.

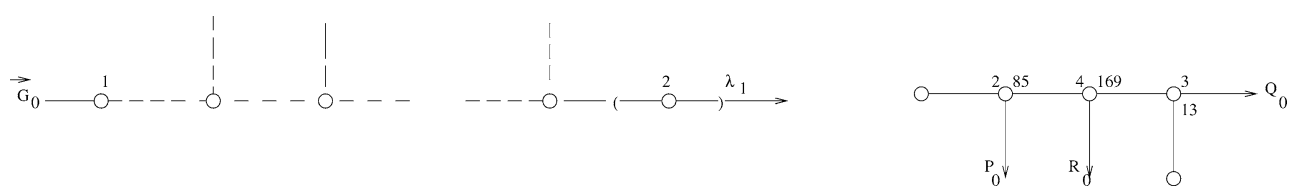

Fig. 24.

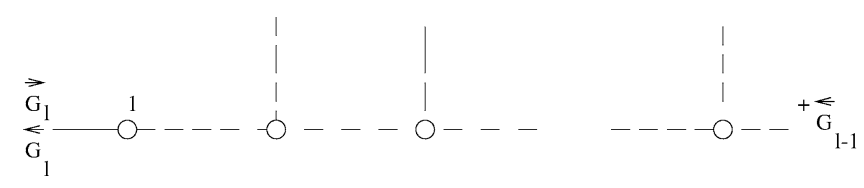

Fig. 25. 
LEMMA A.6. - The splice diagram of the germ $\left\{P_{l}=0\right\} \cup\left\{R_{l}=\mu\right\} \cup\left\{Q_{l}=0\right\}$ is shown on the lefthand side of Fig. 26.

The proof uses the same argument as above. One example of such a rational function is

$$
F_{2}=\phi P_{0}+a Q_{0}^{4}, \quad P=\left(P_{0}^{8}+F_{2}^{5}\right) / Q_{0} .
$$

The polynomial $P$ has degree 38 and is in a $(0,1)$-pencil with $P_{0}$. Its splice diagram is shown on the righthand side of Fig. 26.

Next we recall the graphs that $\mathrm{H}$. Kashiwara gives for the case $\mathcal{F}_{I}$.

CASE 1. $-I(0)$.

Let us consider the graph on the left-hand side of Fig. 27. This produces the splice diagram on the right-hand side of Fig. 27, which fits the case of the two conics mentioned in Example 2.14.

CASE 2. $-I^{+}\left(N ; \lambda_{1}, \ldots, \lambda_{N}\right)$.

In this case we have the graph and the splice diagram shown in Fig. 28.

CASE 3. $-I^{-}\left(N ; \lambda_{1}, \ldots, \lambda_{N}\right)$.

In this case we have the graph and the splice diagram shown in Fig. 29.
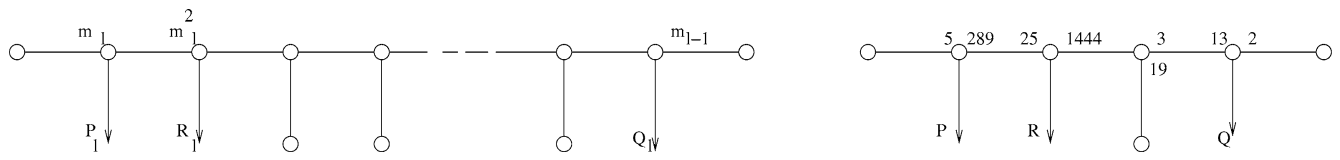

Fig. 26.
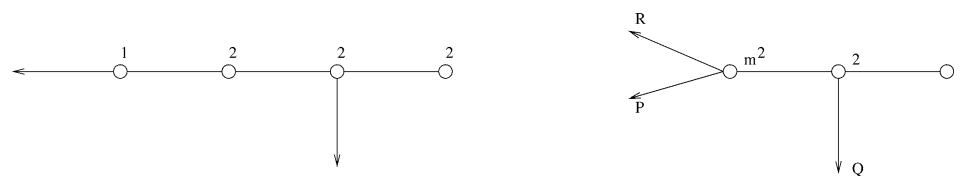

Fig. 27.
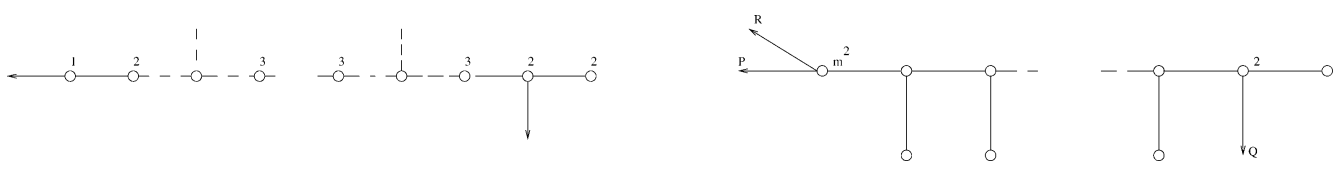

Fig. 28.

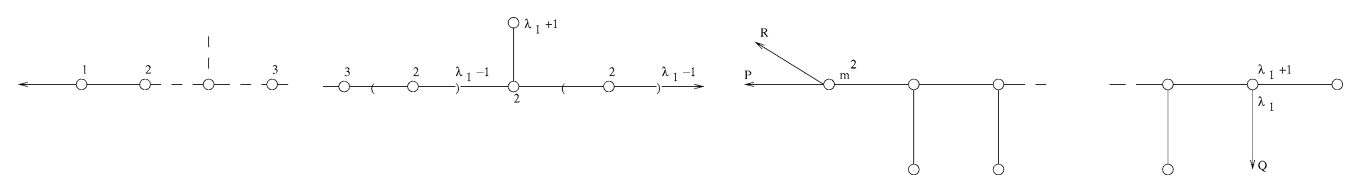

Fig. 29. 


\section{REFERENCES}

[1] A'CAmpo N., La fonction zeta d'une monodromie, Comment. Math. Helv. 50 (1975) 233-248.

[2] Artal Bartolo E., Forme de Jordan de la monodromie des singularités superisolées de surfaces, Mem. Amer. Math. Soc. 109 (525) (1994).

[3] Artal Bartolo E., Sur les couples de Zariski, J. Algebraic Geom. 3 (2) (1994) 223-247.

[4] Artal Bartolo E., Cassou-Noguès Pi., Luengo I., Melle-Hernández A., Denef-Loeser zeta function is not a topological invariant, J. London Math. Soc. (2001), to appear.

[5] Artal Bartolo E., Luengo I., Melle-Hernández A., On a conjecture of W. Veys, Math. Ann. 317 (2) (2000) 325-327.

[6] Denef J., Report on Igusa's local zeta function, in: Astérisque, Vol. 201-203, 1991; Exp. No. 741, Séminaire Bourbaki, Vol. 1990/91, 1992, 359-386.

[7] Denef J., Loeser F., Caractéristiques d'Euler-Poincaré, fonctions zêta locales et modifications analytiques, J. Amer. Math. Soc. 5 (4) (1992) 705-720.

[8] Denef J., Loeser F., Motivic Igusa zeta functions, J. Algebraic Geom. 7 (3) (1998) 505-537.

[9] Denef J., Loeser F., Germs of arcs on singular algebraic varieties and motivic integration, Invent. Math. 135 (1) (1999) 201-232.

[10] Denef J., LOESER F., Geometry on arc spaces of algebraic varieties, to appear in Proceedings of the Third European Congress of Mathematics, Barcelona 2000, p. 22, math.AG/0006050.

[11] Dimca A., On the connectivity of a complex affine hyperserfaces, Topology 29 (1990) 511-514.

[12] Dimca A., NÉMETHI A., On the monodromy of complex polynomials, Duke Math. J. 108 (2) (2001) 199-209.

[13] Eisenbud D., Neumann W.D., Three-Dimensional Link Theory and Invariance of Plane Curve Singularities, in: Annals of Mathematics Studies, Vol. 110, Princeton University Press, Princeton, NJ, 1985.

[14] EsnaUlt H., Fibre de Milnor d'un cône sur une courbe plane singulière, Invent. Math. 68 (3) (1982) 477-496.

[15] Gurjar R., Parameswaran A., Open surfaces with non-positive euler characteristic, Compositio Math. 99 (1995) 213-229.

[16] De Jong A.J., Steenbrink J., Proof of a conjecture of W. Veys, Indag. Math. (N.S.) 6 (1) (1995) 99-104.

[17] Kashiwara H., Fonctions rationnelles de type $(0,1)$ sur le plan projectif complexe, Osaka $J$. Math. 24 (3) (1987) 521-577.

[18] KizUKa T., Rational functions of $\mathbb{C}^{*}$-type on the two-dimensional complex projective space, Tohoku Math. J. (2) 38 (1) (1986) 123-178.

[19] KojIma H., On Veys' conjecture, Indag. Math. (N.S.) 10 (4) (1999) 537-538.

[20] Libgober A., Alexander polynomial of plane algebraic curves and cyclic multiple planes, Duke Math. J. 49 (4) (1982) 833-851.

[21] Loeser F., Fonctions d'Igusa $p$-adiques et polynômes de Bernstein, Amer. J. Math. 110 (1) (1988) $1-21$.

[22] Loeser F., Fonctions d'Igusa $p$-adiques, polynômes de Bernstein, et polyèdres de Newton, J. Reine Angew. Math. 412 (1990) 75-96.

[23] Loeser F., VAquié M., Le polynôme d'Alexander d'une courbe plane projective, Topology 29 (2) (1990) 163-173.

[24] Looijenga E., Motivic measures, Sèminaire Bourbaki, exposé 874, to appear in Asterisque, 2000, p. 25, math.AG/0006220.

[25] Luengo I., The $\mu$-constant stratum is not smooth, Invent. Math. 90 (1) (1987) 139-152.

[26] Rodrigues B., Veys W., Holomorphy of Igusa's and topological zeta functions for homogeneous polynomials, Pacific J. Math., to appear.

[27] SIERSMA D., Variation mappings on singularities with a 1-dimensional critical locus, Topology 30 (3) (1991) 445-469.

[28] Stevens J., On the $\mu$-constant stratum and the $V$-filtration: an example, Math. Z. 201 (1) (1989) 139-144. 
[29] VEYs W., Poles of Igusa's local zeta function and monodromy, Bull. Soc. Math. France 121 (4) (1993) 545-598.

[30] VEYs W., Determination of the poles of the topological zeta function for curves, Manuscripta Math. 87 (4) (1995) 435-448.

[31] VeYs W., Zeta functions for curves and log canonical models, Proc. London Math. Soc. (3) 74 (2) (1997) 360-378.

[32] VEYS W., Structure of rational open surfaces with non-positive Euler characteristic, Math. Ann. 312 (1998) 527-548.

[33] VEYs W., Zeta functions and 'Konsevitch invariants' on singular varities, Canad. J. Math. 53 (4) (2001) 834-856.

[34] Yoshihara H., On plane rational curves, Proc. Japan Acad. Ser. A Math. Sci. 55 (4) (1979) 152-155.

(Manuscript received November 28, 2000;

accepted, after revision, November 12, 2001.)

\title{
E. Artal Bartolo
}

Departamento de Matemáticas,

Universidad de Zaragoza,

Campus Plaza San Francisco s/n,

E-50009 Zaragoza, Spain

E-mail: artal@posta.unizar.es

\section{P. CASSOU-NOGUÈS}

Laboratoire de mathématiques pures,

Université Bordeaux I, 350,

Cours de la Libération,

33405 Talence Cedex, France

E-mail: cassou@math.u-bordeaux.fr

\author{
I. Luengo and A. Melle HeRnÁndez \\ Departamento de Álgebra, \\ Universidad Complutense, \\ Ciudad Universitaria $\mathrm{s} / \mathrm{n}$, \\ E-28040 Madrid, Spain \\ E-mail: iluengo@eucmos.sim.ucm.es, \\ amelle@eucmos.sim.ucm.es
}

